\title{
The Society for Social Medicine, 46th Annual Scientific Meeting: Abstracts
}

J. Epidemiol. Community Health 2002;56;4-29 doi:10.1136/jech.56.suppl_2.ii4

Updated information and services can be found at:

http://jech.bmjjournals.com

These include:

Rapid responses You can respond to this article at:

http://jech.bmjjournals.com/cgi/eletter-submit/56/suppl_2/ii4

Email alerting Receive free email alerts when new articles cite this article - sign up in the box at service the top right corner of the article

Notes 


\section{Plenary sessions}

\section{PRIMARY CARE GEOGRAPHY THE CONUNDRUM OF RESPONSIBLE OR RESIDENT POPULATIONS}

R.C. Wilson. Department of Public Health and Epidemiology, University of Birmingham, Birmingham, B15 2TT

Introduction: The Public Health geography of the new NHS is Primary Care based with responsibility devolved to Primary Care Trusts by "Shifting the Balance of Power". This sets a statistical conundrum, whether to use a Primary Care/GP relevant list based view, a population that may not respect administrative boundaries or the traditional resident based view. It has been stated that the list based view is appropriate for the management of Primary Care resources, whereas for the wider issues of public health, such as inequalities, the resident view is more appropriate.

But does it really matter which is used? This paper seeks to address that question looking at activity patterns for the two populations.

Methods: Hospital admissions data for the West Midlands region was supplied by the Department of Health for the year 1999/2000, with a Primary Care Group (PCG) code allocated on the basis of the patient's registered GP. This code was used to create the Responsible Population. The data were postcode matched to PCG boundaries built up from electoral wards. This gave the Resident Population, patients who were resident within the PCG but who may not be registered there.

Results: There were 61 PCGs across the West Midlands; however West Midlands residents were registered with 429 PCGs. A PCG's responsible population contained patients registered with between 22 and 81 (median 43) other PCGs. This equated to between $31.1 \%-96.6 \%$ (mean $70.7 \%$ ) of a PCG's responsible admissions being from its resident population. There are considerable differences for specific conditions, by demography and deprivation.

Conclusion: The scale of dispersion of patients cannot be ignored. Commissioners and managers have to be aware that some Primary Care organisations may have little ability to influence the health of their locality when more than $30 \%$ of the population for whom they are commissioning are not on their lists. This is complicated further by the non-random nature of the dispersion, as there are systematic variations.

This research poses a number of challenges to both policymakers and commissioners. Are the traditional monitoring tools appropriate? Based on residency, Health Authorities had an average crossboundary flow of between $5-7 \%$ and their responsibilities were clearly defined by boundaries, where as cross boundary flow at primary care level is six times higher making many boundaries meaningless. The implications of this will be discussed.

\section{INTELLIGENCE IN CHILDHOOD AND MORTALITY, MIGRATION, QUESTIONNAIRE RESPONSE RATE, AND SELF-REPORTED MORBIDITY AND RISK FACTOR LEVELS IN ADULTHOOD: PRELIMINARY FINDINGS FROM THE ABERDEEN 'CHILDREN OF THE 1950s' STUDY}

G.D. Batty', H. Clark², S.M.B. Morton', D. Campbell², S. Macintyre ${ }^{3}$, M. $\mathrm{Hall}^{2}$, D.A. Leon'. 'Epidemiology Unit, London School of Hygiene and Tropical Medicine, London; ' Dugald Baird Centre, University of Aberdeen, Aberdeen; ${ }^{3}$ MRC Social and Public Health Sciences Unit, University of Glasgow, Glasgow

Objective: To explore the relation of intelligence quotient (IQ) at 7 years of age to all-cause mortality, migration, questionnaire response rate, and self-reported morbidity and risk factor levels in adulthood.

Design: Retrospective cohort study.

Setting: Aberdeen, UK.

Participants: 12,150 children (6,276 boys; 5874 girls) born from

1950-55 and in primary school in December 1962.

Main outcome measures: All-cause mortality, migration, questionnaire response rate, and self-reported morbidity and risk factor levels in adulthood.

Results: The whereabouts and vital status of $97.6 \%(11,859)$ of subjects at $45-50$ years of age were ascertained using the NHS Central Register; $3.7 \%$ (454) had died. IQ measured at 7 years of age was inversely associated with all-cause mortality rates in adulthood, such that a greater mortality rate was seen in adults who, as children, had low IQ test scores ( $\leqslant 89$ vs. $\geqslant 120$; age-adjusted hazards ratio $1.89 ; 95 \%$ confidence interval 1.4 to 2.6$)$. The magnitude of this relationship was essentially unaltered on adjusting for paternal occupational social class at birth. In comparison to children with low $I Q$ test scores, children with high scores were more likely to move away from Aberdeen to other areas of Scotland, other parts of the UK, and abroad.

In a questionnaire survey of surviving Scottish residents (response rate $62.9 \% ; \mathrm{N}=6039$ ) we enquired about morbidity and risk factor levels. Response rate was directly related to IQ at age 7 across the full range of scores. IQ was inversely and incrementally related to the prevalence of long-term illness/disability, poor self-perceived health, and feelings of depression/unhappiness. IQ was also associated with a range of behavioural, socio-economic, psycho-social, and anthropometric risk factors such as cigarette smoking, inebriationinducing levels of alcohol consumption, educational attainment, income, housing tenure, car ownership, social support, and height, with the most favourable levels seen in the adults who, as children, had recorded high scores on the IQ test. These associations held after adjusting for paternal occupational social class at the birth of the subject.

Conclusions: In this follow-up of Aberdeen primary school children, IQ score was inversely related to adult all-cause mortality rates and strong relationships were also seen with a range of health, behavioural, socio-economic, psycho-social, and anthropometric indices in adulthood, independent of socio-economic position at birth. These findings suggest that childhood $I Q$ score is capturing an individual-level characteristic that is predictive of adult disease risk.

\section{HIGH RISK SEXUAL BEHAVIOUR INCREASES AMONG LONDON GAY MEN BETWEEN 1998-2001: WHAT IS THE ROLE OF HIV OPTIMISM?}

J. Elford', G. Bolding', L. Sherr'2. 'City University London, Institute of Health Sciences \& St Bartholomew School of Nursing and Midwifery; ${ }^{2}$ Royal Free and University College Medical School, London

Objective: It has been suggested that the recent increase in high risk sexual behaviour among gay men is due to optimism in the light of new HIV drug therapies (HIV optimism). There is, however, little sound evidence to support this hypothesis. The objective of this study was examine whether HIV optimism can indeed account for the recent increase in high risk sexual behaviour among London gay men using data collected over a four year period.

Methods: Gay men ( $\mathrm{n}=2938$ ) using central London gyms were surveyed annually between 1998-2001. Each year information was collected on HIV status, unprotected anal intercourse (UAI) in the previous 3 months and agreement/disagreement with two optimism statements "I am less worried about HIV now that treatments have improved", "I believe that new drug therapies make people with HIV less infectious". Those who agreed were classified as "optimistic".

Results: Between 1998-2001 the percentage of men reporting high risk UAI (ie UAI with a casual partner of unknown or discordant HIV status) increased: HIV positive men, $15.3 \%$ to $38.8 \%, p<0.001$; HIV negative men, $6.8 \%, 12.1 \%, p<0.01$; never-tested men $2.1 \%$, $7.7 \%, p<0.01$. Overall, less than a third of the men were classified as being optimistic. In cross-sectional analysis, optimistic HIV positive and negative men (but not the never-tested) were more likely to report high risk UAI than other men $(p<0.05)$. However, the increase in high risk UAl between 1998-2001 was seen in both those who were optimistic (the minority) and those who were not (the majority) $(p<0.05)$. In multivariate analysis, the modelled increase in high risk UAl over time remained significant after controlling for HIV optimism $(p<0.001)$ with no significant interaction between optimism and time $(p>0.05)$. For HIV positive men, the adjusted odds ratio for year of survey was $1.4(95 \% \mathrm{Cl} 1.2,1.6, \mathrm{p}<0.001)$ ie the risk of reporting high risk UAI increased by $40 \%$ each year after controlling for HIV optimism. For HIV negative men the adjusted OR was 1.5 (1.2, 2.0, $\mathrm{p}<0.001)$.

Conclusion: Among London gay men, no difference was detected between those who were optimistic and those who were not in the rate of increase in high risk sexual behaviour between 1998-2001. Our findings suggest that HIV optimism is unlikely to explain the recent increase in high risk sexual behaviour in these men. Identifying the 
factors which may underlie this increase should now be given the utmost priority.

\section{IS 'WATCHFUL WAITING' A REAL CHOICE FOR MEN WITH PROSTATE CANCER? A QUALITATIVE STUDY}

S. Ziebland', A. Chapple, A. McPherson, A. Herxheimer, S. Shepperd, R. Miller2. 'Cancer Research UK, General Practice Research Group, Department of Primary Health Care University of Oxford; ${ }^{2}$ DIPEx Department of Primary Health Care, University of Oxford

Treatment for prostate cancer is highly controversial because no randomised, controlled trials have shown whether or not active intervention increases survival. Watchful-waiting, also known as active surveillance, or monitoring, implies no therapy once the disease has been diagnosed until the patient has some symptom resulting from complications of local or systemic disease. The aim of this paper is to explore what leads men to choose 'watchful waiting' rather than active treatment for cancer of the prostate.

Method: We interviewed 50 men with confirmed prostate cancer, in England, Wales and Scotland, about all aspects of their illness, as a study for the DIPEx (www.dipex.org) website. The sample included men at different stages of diagnosis, with experience of a wide range of treatments. In- depth interviews were conducted in men's homes, tape recorded and transcribed for qualitative thematic analysis.

Results: Watchful waiting would have been clinically inappropriate for those men with serious urinary symptoms and those with metastatic disease (almost half the sample). However, few of the men who might have chosen watchful-waiting remembered this being presented as a serious option. Most in this group chose radical prostatectomy, radiotherapy, brachytherapy or cryosurgery. The few who chose watchful waiting had found doctors who supported their decision, had assessed the evidence from Internet sites, and were concerned about the side effects and uncertain outcome of treatment. Men who chose watchful waiting, as well as those who opted for treatment, described considerable pressure from family members, doctors or support groups, to seek active treatment. Concerns included that they should 'do something' when faced with a cancer diagnosis and that they might be too old to be allowed treatment if and when it proved necessary.

Conclusion: This study helps to explain why some men will not contemplate 'watchful waiting', and why others may find it difficult to pursue that option. The popular discourse that all cancers must be 'fought' with a positive attitude and aggressive treatments contributes to the difficulties that men with prostate cancer have in choosing this approach, despite the lack of evidence that active treatment will be beneficial.

\section{URBAN-RURAL DIFFERENCES IN THE OCCURRENCE OF DEPRESSIVE DISORDER IN EUROPE: EVIDENCE FROM THE ODIN STUDY}

V. Lehtinen', C. Dowrick², E. Michalak ${ }^{3}$, C. Wilkinson ${ }^{3}$, J.-L. AyusoMateos $^{4}$, O.S. Dalgard ${ }^{5}$, P. Casey ${ }^{6}$, J.-L. Vázquez-Barquero ${ }^{4}$, G. Wilkinson ${ }^{2}$ and The ODIN Group 'STAKES, Finland; 'University of Liverpool; " ${ }^{2}$ niversity of Wales College of Medicine; ${ }^{4}$ University of Cantabria; ${ }^{5}$ University of Oslo; ${ }^{\circ}$ University College Dublin

Background: The majority of previous research on the prevalence of depression has been conducted in urban areas. Studies comparing urban-rural differences in this respect have given contrasting results, at least partly due to methodological inconsistencies.

Aim: To analyse the factors which might explain the urban rural differences in the occurrence of depressive disorder across five urban and four rural areas in Europe.

Method: A representative sample of 14387 people aged between 18 and 64 residing in specified urban and rural areas were screened by the Beck Depression Inventory for depressive disorder. Those over cut-off and a $5 \%$ random sample of those under cut-off underwent diagnostic interview including the SCAN version 2.0, and completed a battery of additional research instruments.

Results: The estimated one-month prevalence of depressive disorder according to ICD-10 was $9.0 \%$ in the total ODIN sample. A large between country variation was found in female urban prevalence, with Ireland and UK having a remarkably high rate. The women in these same countries showed a significant urban/rural difference, whereas in men and in the total sample this difference was non-significant. Multivariate analyses was undertaken including the following factors: country, age, place of birth, marital status, employment status, living alone/with others, availability of practical help, presence of confidants, recent negative life events, urban/rural setting. The significance of urban/rural setting as an explanation for differences in depression in women disappeared on this analysis. The most important determinants explaining the risk of depressive disorder in women were: having difficulties in getting practical help from neighbours, lacking a confidant, experiencing two or more threatening life events during the previous six months, being separated or widowed, and belonging to the middle age group.

Conclusions: ODIN is the first European study on occurrence of depressive disorder in both urban and rural settings, allowing close analysis of urban/rural differences. Variation in sample sizes indicates a need for caution in interpreting results. However the most striking result was the large urban/rural difference in women in UK and Ireland, which was mainly explained by variation in social support.

\section{ASSOCIATION BETWEEN INFANT NUTRITION AND BLOOD PRESSURE IN EARLY ADULTHOOD: THE BARRY CAERPHILLY GROWTH COHORT STUDY}

R.M. Martin', A. McCarthy', D.P. Davies, G. Davey Smith', Y. Ben-Shlomo'. 'Department of Social Medicine, University of Bristol; ${ }^{2}$ Department of Child Health, University of Wales College of Medicine

Objective: To determine whether early infant nutrition, as measured by intake of dried formula milk in the first 3 months of life, is associated with blood pressure in early adulthood.

Design: Long-term follow-up (1997-1999) of a randomised controlled trial (1972-1974) into which mothers and their offspring were randomised to milk supplementation or usual care (Barry Caerphilly Growth (BCG) study).

Setting: The towns of Barry and Caerphilly in South Wales.

Participants: 679 males and females aged between 23 and 27 years at follow-up.

Main outcome measures: Systolic and diastolic blood pressure.

Results: Subjects who were followed up were similar to those who were not for a range of social and demographic factors. There was a clear positive dose response relationship between dried milk intake at 3 months of age and systolic blood pressure in early adulthood $(p=0.005)$ : the mean systolic blood pressure was $120.2 \mathrm{mmHg}$ versus $114.0 \mathrm{mmHg}$ in the highest versus lowest quartiles of intake. Dried milk intake at 3 months of age was positively associated with diastolic blood pressure (highest versus lowest quartile: $71.2 \mathrm{mmHg}$ vs 68.9 $\mathrm{mmHg} ; p=0.059$ ). In multivariable linear regression models adjusted for sex, intervention group, birthweight z-scores (standardised for sex and gestational age), social class in childhood, age at follow-up, alcohol and smoking, intake of dried milk was positively associated with systolic and diastolic blood pressure. For each increase in quartile of dried milk intake (ounces) there was a $1.28 \mathrm{mmHg} 195 \% \mathrm{Cl}$ : 0.46 to 2.10$)$ increase in systolic and a $0.63 \mathrm{mmHg}(0.04$ to 1.22$)$ increase in diastolic blood pressure. These coefficients were attenuated when adult BMI and height were included in the models, but the association of dried milk intake with systolic blood pressure remained significant (1.07; 0.27 to 1.87). The association of dried milk intake and systolic blood pressure was stronger in males than females ( $p$ for interaction: 0.05).

Conclusions: Our results are consistent with the hypothesis that high blood pressure in later life is influenced by early postnatal nutrition independent of birthweight. The public health implications of our findings are important, since reductions in population mean blood pressure levels observed in this study would be predicted to be as or more effective in reducing blood pressure-related morbidity or mortality than targeting interventions at high-risk individuals only. The suggestion of postnatal nutritional influences on adult health offers the possibility that interventions to optimise infant nutrition may have important long-term health benefits.

\section{Inequality I}

\section{COMPARING THE HEALTH STATUS OF URBAN AND RURAL POPULATIONS}

K.A. Levin. MRC Social and Public Health Sciences Unit, University of Glasgow, 4 Lilybank Gardens, Glasgow G12 8RZ

Background: Previous research suggests that there are significant differences in health between urban and rural areas. Recent reports cast doubt on the reliability of earlier findings that the population living in 
urban areas is less healthy than that in rural areas. Deprivation indicators have been criticised for being biased towards urban areas, while rurality has been classified on a number of different scales.

Objectives: To describe the pattern and magnitude of urban/rural variation in health in Scotland using limiting long term illness (LLTI) as a health indicator and to examine the factors associated with health inequalities in urban and rural areas.

Data and methods: Postcodes classified using the Scottish Household Survey rurality indicator were aggregated and reclassified using clustering techniques to give a rurality indicator at postcode sector level (approximate average population 5000). Premature and all-age standardised illness ratios (SIRs) based on LLTI as reported in the 1991 census were investigated for each rurality. A variety of socio-economic determinants of LLTI (again taken from the census) were investigated using multilevel Poisson regression.

Results: The areas with the highest SIRs $(>125)$ are predominantly urban (78:5 urban:rural sectors) whilst the lowest $(<75)$ are found in both urban and rural areas $(26: 35)$. Variation in SIRs within rural areas is generally smaller than within urban areas. Areas of small populations, within a 30-minute drive to large urban settlements, have the least LLTI. Determinants of illness vary according to type of rurality. The total variation in the models used to describe urban and suburban areas and remote towns is reduced by $84-89 \%$ when socio-economic factors are included. Areas composed of accessible settlements of less than 3000 people see a reduction of $56 \%$, whilst similarly sized settlements in remote areas see a $35 \%$ reduction of variation due to the inclusion of socio-economic factors.

Conclusions: Models for areas comprising accessible and/or remote villages show few of those socio-economic factors considered to be significantly associated with illness. This suggests that either the explanatory variables used are not appropriate for modelling illness in rural communities or that these areas are so heterogeneous that further subdivisions of area type are required for the more remote, less populated sectors. This is further supported by the relatively small reduction in total variation in these models once socio-economic factors are included.

\section{AGE-SPECIFIC DIFFERENCES IN AREA MORTALITY EFFECTS}

A.H. Leyland. MRC Social and Public Health Sciences Unit, University of Glasgow

Introduction: Areas with high mortality at one point in time tend to have high mortality subsequently. This paper investigates whether the (all cause) mortality of a certain age group living in a certain area is best predicted by the previous mortality experience of people of the same age in that area (an age effect), the previous mortality experience of people of that birth cohort in that area (a cohort effect), or the mortality of other ages in that area at that time (a general malaise associated with the area)?

Data and methods: All cause mortality and population data for all 459 government districts in GB were analysed in 5-year age bands for the periods 1979, 1984, 1989 and 1994. Age- specific mortality rates were estimated in each area for each band between the ages of 0-84. Age-exclusive mortality rates (the age standardised mortality rate among all other age bands) were also estimated. Mortality rates were estimated for 1984, 1989 and 1994 using the age-specific mortality rates from the previous period relating to the same age group and the same cohort, and the age-exclusive mortality rates from the same period. All analyses were conducted using multilevel Poisson regression.

Results: The variation between areas in mortality rates is generally largest among the younger age groups and tends to decrease with increasing age. For those aged 45-84 the mortality rate among other age groups in the area consistently explains the largest proportion of the variation between areas. The proportion of variation explained is lowest amongst the $80-84$ age group (54\%-63\% over the 3 periods) and highest among the $65-69$ age group (93\%-98\%). For those aged 25-34 the mortality rate in the same age group in the area 5 years previously consistently explains the largest proportion of the variation between areas. A lower proportion of the variation is explained than among the older age groups (range 33\%-93\%). The results for other age groups are inconclusive.

Conclusions: Variations in mortality are greater among younger age groups. Areas with high mortality rates for young adults (25-39) are not necessarily those areas with high mortality rates among other age groups; however, certain areas do tend to have consistently high mortality rates in this age group. This will in part reflect the lack of correlation within areas between mortality rates for the younger and older age groups, a fact which is masked by age standardisation.

\section{ENVIRONMENTAL AND SOCIO-ECONOMIC HEALTH EQUITY IN ENGLAND AND WALES}

B.W. Wheeler, Y. Ben-Shlomo, E. Whitley. Department of Social Medicine, University of Bristol, UK

Objective: To investigate environmental (in)equity and its role in determining socio-economic health inequalities.

Design: Ecological/cross-sectional studies.

Setting: England and Wales.

Participants: Population 1991 (42,972,952); Health Survey for England 1994-97 (71,471).

Main outcome measures: Small-area indices of potential environmental health risk (ambient air quality, licensed industrial atmospheric chemical releases, landfills and registered Control Of Major Accident Hazard sites). Ecological: mortality due to ischaemic heart disease, chronic obstructive pulmonary disease, lung cancer and all-causes, 1991-95. HSE: all-cause mortality; limiting long-term illness; asthma; lung function.

Results: Environmental Equity: Positive associations were found between deprivation measures and environmental indices. An urban census ward in the most deprived $20 \%$ compared to one in the least deprived $20 \%$ (Carstairs Index) was 6.7 [95\% Cl 4.8-9.3] times as likely to be within $1 \mathrm{~km}$ of a large atmospheric release site, 1.6 [1.22.0] times as likely to be within $1 \mathrm{~km}$ of a landfill and 4.0 [3.1-5.0] times as likely to be within $1 \mathrm{~km}$ of a COMAH-registered site. Associations persisted in rural areas, but were generally weaker. The relationship between socio-economic status and the ambient air quality index is more complex, with associations positive in urban areas and negative in rural areas.

Health outcomes: Ecological analyses demonstrated small adverse effects of increased environmental hazard on mortality. Effect estimates were mostly attenuated when adjusted for deprivation. Analysis of individual HSE data produced complementary results, with the most apparent effect being that of ambient air pollution on lung function. Residence in the $20 \%$ of wards with highest levels of ambient air pollution compared to the lowest $20 \%$ was associated with a reduction of $8.3 \%$ [4.9-1 1.7] in boys', and $4.5 \%$ [0.9-8.2] in girls', mean height-adjusted $\mathrm{FEV}_{1}$, after adjustment for social class of head of household, passive smoke exposure and urban-rural status. Socioeconomic health gradients were slightly attenuated by adjustment for environmental measures.

Conclusions: Standard small-area indicators of deprivation are strongly related to small-area indices of potential environmental health risk. These indices have measurable adverse effects on some health outcomes after adjustment for socio-economic status and may, to a small extent, explain gradients between area deprivation and morbidity/mortality. Associations are complex, appear to interact with urban-rural status and are subject to methodological limitations, such as errors due to spatial autocorrelation. The study raises issues of environmental inequity and has implications in terms of social justice and health, environmental and planning policy.

\section{LIFE EXPECTANCY BY SOCIO-ECONOMIC STATUS}

\section{A. Donkin, P. Goldblatt, K. Lynch. Office for National Statistics, London}

Objective: To consider the use of the ONS Longitudinal Study to monitor socio-economic inequalities in health following the introduction of the National Statistics Socio-Economic Classification (NS SEC).

Design: Longitudinal study.

Setting: England and Wales.

Participants: $1 \%$ representative sample of the population of England and Wales since the 1971 Census, (approximately 800,000 study members).

Main outcome measure: Life expectancy.

Results: In January 2001 the Office for National Statistics published life expectancy figures for males and females for 1997-99 by social class. The figures showed that the differential between life expectancy at birth for men in social class I (professionals) and social class $V$ (unskilled workers) had narrowed to 7.4 years, from 9.5 years in 1992-96. These life expectancy calculations required age-specific mortality rates by social class at all ages, not just at working ages. This presentation explains how social class was attached to those of working and non-working age and discusses the advantages and disadvantages of the method. To illustrate this discussion three sets of life expectancy figures for 1997-99 are calculated based on social class attached at or after the 1971, 1981 and 1991 Censuses, respectively.

The life expectancy figures calculated using social class at or after 1991 are less reliable than those calculated using earlier starting 
points, particularly for females. Three-quarters of female deaths could not be linked to a social class as a result of the ageing population. This poses a particular challenge in presenting trends by NS SEC rather than social class. NS SEC is not available on data prior to 2000 , thus it would be necessary to attach an approximation of NS SEC at the earliest possible time point to calculate trends in life expectancy. An approximation to NS SEC based on data available between 1990 and 2000 has been developed (NS SEC90). Ninety percent of deaths in 2000 were correctly classified to the same NS SEC and NS SEC90 category. The implications of using NS SEC90 for life expectancy calculations and approximating to NS SEC prior to 1990 are discussed.

Conclusions: This paper highlights the difficulties associated with presenting trends by NS SEC rather than social class using life expectancy as an indicator. Possible solutions are discussed.

\section{Risk factors}

\section{HELICOBACTER PYLORI INFECTION AND CORONARY HEART DISEASE IN THE CORK 5C STUDY: A POSSIBLE LINK BETWEEN INFECTION AND INFLAMMATION?}

S. O' Sullivan, J. Sheehan, S. Neilson, R. Hinchion, I.J. Perry. Department of Epidemiology and Public Health, University College Cork, Ireland

Introduction: Coronary heart disease (CHD) is one of the leading causes of mortality in Western countries. The incidence of cardiovascular disease in Ireland is among the highest in Europe. While major risk factors for CHD are well known, they do not fully explain all of causative factors of the disease. Evidence exists to suggest that active inflammation and/or chronic infection may play a role in CHD. Helicobacter pylori infection usually acquired in childhood has been associated with an increased risk of CHD.

Objective: To investigate the association between acute coronary syndrome and $\mathrm{H}$. pylori infection in an Irish population sample.

Design: A community based case-control study.

Methods: The Cork $5 \mathrm{C}$ study is a community based case control study. Cases were first event myocardial infarction or unstable angina, aged between 35 and 74 years, who were admitted to the coronary care units in the Cork area. AMl and first event angina were defined on the basis of standard internationally acceptable criteria. Controls were randomly sampled from the age sex registers or practice lists of the general practices from which the patients were referred. Both cases and controls underwent standardised assessment, including questionnaires, physical measurements and blood tests. H. pylori infection was diagnosed serologically (with lgG titers $>1.8$ seropositive) and the association between infection and CHD was estimated by binary logistic regression.

Results: 227 cases and 277 controls were recruited giving a response rate of $97 \%$ and $73 \%$ respectively. The mean age of cases and controls was $59.30 \%$ of cases and $35.3 \%$ of controls were seropositive for $\mathrm{H}$. pylori infection. The unadjusted Odds Ratio for $\mathrm{H}$. pylori infection between cases and controls was 1.03 (95\% C.I. 0.95 1.11). On adjustment for known cardiovascular risk factors, age, sex, body mass index, smoking, and cholesterol the adjusted Odds Ratio was for $\mathrm{H}$. pylori infection between cases and controls was $1.06195 \%$ C.I. 0.90-1.26). There was no significant difference between socioeconomic group, smoking status and $\mathrm{H}$. pylori status.

Conclusion: These data provide no support for the hypothesis linking $\mathrm{H}$. pylori infection to acute coronary syndromes. The positive results in previous studies may have been due to selection bias or confounding.

\section{SUPPRESSED VERSUS EXPRESSED ANGER AND RISK OF CORONARY HEART DISEASE AND STROKE: THE CAERPHILLY STUDY}

M. May', S. Ebrahim', S. Stansfeld ${ }^{2}$, J. Gallacher ${ }^{3}$, G. Davey Smith'. 'Department of Social Medicine, University of Bristol, Canynge Hall, Whiteladies Road, Bristol BS8 2PR; ${ }^{2}$ Department of Psychiatry, Queen Mary \& Westfield College, University of London, Mile End Road, London E1 4NS; ${ }^{3}$ Department of Epidemiology \& Public Health, University of Wales College of Medicine, Heath Park, Cardiff CF14 4XN

Objectives: To investigate the relationship between personality trait and all-cause, cardiovascular causes of mortality, coronary heart disease events and stroke events. In particular, whether Type A coronary prone behaviour (measured by Framingham Type A, Jenkins Type A and Bortner questionnaires), anxiety (Spielberger Self-Evaluation questionnaire) and different ways of managing anger, that is, tendency to suppress or express anger (Framingham Anger Scales) are associated with disease outcomes.

Design: Population cohort study with 15 years follow up.

Participants: 2398 men aged 49-64 years who participated in phase II of the Caerphilly study in 1984 to 1988.

Main outcome measures: All cause mortality, cardiovascular mortality, coronary heart disease and stroke events.

Methods: Summary scores were calculated from the questionnaires. Subjects were divided into quintiles of score for each psychological measure. The odds ratio of each outcome was calculated per quintile change in psychological measure.

Results: 561 (25.3\%) men died, 237 (10.7\%) died from CVD, $288(13 \%)$ suffered a CHD event and $144(6.5 \%)$ had a stroke. We found no association between Type A personality measures or anxiety trait and mortality or CHD or stroke. Suppressed anger was not associated with mortality [OR $0.9995 \% \mathrm{Cl}(0.92$ to 1.06$)$ per quintile] or CHD events [OR $0.99(0.88$ to 1.11$)]$, but was strongly associated with stroke events [OR $1.44(1.11$ to 1.86$)]$. In contrast, those who expressed anger had a lower odds ratio per quintile for all cause mortality [OR $0.88 \quad(0.77$ to 1.00$)$ ] which was attributable to cardiovascular death [OR 0.79 (0.65 to 0.97)]. The OR per quintile of expressed anger for CHD events is similar to CVD mortality[OR $0.76(0.60$ to 0.96$)]$, but there is no decrease of OR with stroke events [OR $1.04(0.68$ to 1.58)]. These results were not attenuated by adjusting for standard CVD risk factors.

Conclusions: Type A behaviour and anxiety do not predict mortality, CHD or stroke in this cohort. However, questions designed to show how subjects react when angry show a strong pattern of association with disease outcomes. Subject who suppress anger are more likely to suffer a stroke, but do not have a raised risk of CHD. In contrast to this, subjects who express anger are less likely to suffer CVD mortality or CHD events, but have average risk of stroke.

\section{INFLUENCE OF HEIGHT, LEG AND TRUNK LENGTH ON PULSE PRESSURE}

C. Langenberg, D. Kuh, R. Hardy, M.E.J. Wadsworth. MRC National Survey of Health and Development, Department of Epidemiology and Public Health, UCL Medical School, London

Background: Children who grow up under adverse socioeconomic circumstances have shorter height and legs compared to their peers from higher social backgrounds, similarly they are at higher risk of cardiovascular disease (CVD). Short height is associated with an increased risk of CVD and the reasons for this relationship remain largely unknown. Increased pulse pressure, as the difference between systolic and diastolic blood pressure, has been shown to be an important risk factor for CVD in men and women over 50 years and is determined by the timing of arterial wave reflection, which occurs earlier in people of shorter height.

Objective: To compare the effects of height, leg and trunk length on pulse pressure, systolic and diastolic blood pressure in men and women.

Design: Prospective, population based, birth cohort study.

Setting: England, Scotland and Wales.

Participants: 1472 men and 1563 women born in 1946.

Main outcome measures: Pulse pressure, systolic and diastolic blood pressure at age 53 years.

Results: Pulse pressure increased linearly $(p$ for trend $\leqslant 0.001$ in each case) with decreasing height and leg length in men and women (shortest compared with the longest leg length group, men 55.8 versus $50.7 \mathrm{mmHg}$, women 53.3 versus $48.6 \mathrm{mmHg}$ ). Adjustment for adult confounding factors and childhood social class or birth weight did not attenuate these results $(p \leqslant 0.001)$, except for height in men ( $p=$ 0.01 ). Systolic, but not diastolic blood pressure showed similar, but weaker trends in men and women, except for the association with height in men, which lost statistical significance after adjustment. Trunk length was not associated with any outcome measure in men or women.

Conclusions: Short height and leg length are associated with increased pulse pressure, but not diastolic blood pressure, in middle aged men and women. Pulse pressure is a potential mediator between short height and leg length and increased risk of CVD. Future studies of the association between shortness and CVD should consider measures of arterial hemodynamics, such as pulse pressure. 


\section{HEIGHT, LEG LENGTH AND RISK OF ASYMPTOMATIC PROSTATE CANCER}

S.E. Oliver', J. Holly², J. Donovan', T.J. Peters ${ }^{3}$, R. Persad², D. Gillatt², F. Hamdy', D.E. Neal ${ }^{5}$, D. Gunnell'. 'Department of Social Medicine; ${ }^{2}$ Division of Surgery; ${ }^{3}$ Primary Health Care, University of Bristol; ${ }^{4}$ Academic Urology Unit,University of Sheffield; ${ }^{5}$ School of Surgical Sciences, University of Newcastle

Background and objective: Greater height in adulthood has consistently been associated with increased risk of developing cancer, including prostate cancer. However, there has been little research exploring the relationship between the components of adult height (leg and trunk length) and cancer risk. As the relative velocity of growth in legs and trunk varies with age any such relationships might elucidate periods in the life course of particular significance for cancer development. We therefore used a population-based case-finding exercise recruiting individuals with prostate cancer to a trial of treatment to examine the relative contribution of leg and trunk length to prostate cancer risk.

Methods: A nested case-control study was conducted amongst 7,383 asymptomatic men (50-69yrs) who underwent a prostatespecific antigen (PSA) test as part of the ProtecT study. Anthropometric measures (standing and sitting height and weight) were taken by trained staff in three UK centres. Men with a raised PSA ( $\geqslant 3 \mathrm{ng} / \mathrm{ml}$ ) were offered prostate biopsy. Cases were classified as either localised to the prostate gland (TNM stage $\leqslant T 2$ ) or advanced ( $\geqslant T 3$ ). Leg and trunk length (and leg:trunk ratio) were derived and standardised $z$-scores established for height, leg-length and leg:trunk ratio. Associations between anthropometry and prostate cancer risk were explored using logistic regression, odds ratios were calculated for a one standard deviation increase in each measure.

Findings: A total of 224 men with prostate cancer were identified, $166(74 \%)$ had localised disease. Anthropometry was obtained on $170(76 \%)$ cases. In age-adjusted analyses a weak association was observed between greater adult height and increased risk of cancer (OR $1.08,95 \% \mathrm{Cl} 0.92-1.27$ ). Of the components of height, it was leg length (OR 1.10, 0.94-1.28) rather than trunk length (OR 1.02, $0.87-1.18)$ that accounted for this association. The risk of cancer was greater in men whose legs were longer in relation to their overall height (OR 1.08, 0.93-1.25). This pattern of associations was maintained when analyses were limited to advanced cases and in fully-adjusted models accounting for age, study centre, social class and smoking history.

Interpretation: This study found evidence of a weak relationship between height and prostate cancer risk, consistent in magnitude with previous research but not reaching conventional statistical significance. The finding that leg, rather than trunk length, is the component of height associated with prostate cancer provides some support for the hypothesis that environmental exposures before puberty, when long bones are growing fastest, may have a role in establishing disease risk.

\section{HSR I}

\section{DEAD EASY?: AN ANALYSIS OF THE PRODUCTION OF DEATH CERTIFICATES AND SUGGESTIONS FOR CHANGE}

A. Clarke', J. Gladwin', C. Rooney2', S. Carter', N. Fulop'. 'Health Services Research Unit, London School of Hygiene \& Tropical Medicine, Keppel Street, London, WCIE 7HT; ${ }^{2}$ Office for National Statistcs, 1 Drummond Gate, London, SWIV 2QQ

Introduction: The research reported here was commissioned in the light of the Shipman and other inquiries and alongside moves to rationalise death certification:

Objectives: to undertake a literature review and interviews with key stakeholders to:

Identify the separate roles which a death certificate fulfils.

Identify the processes by which composite data are produced from death certificates, including flow of information and disposal of the body.

Identify the separate roles of the coroner, the registrar and the certifying doctor in the process of certification.

Assess the benefits of a new role: "the medical examiner" to improve the processes and value of death certification.

Methods: Reviews of the literature and Interviews with key stakeholders; ONS, Thames Cancer, Royal College of Pathologists and practising certifying doctors, funeral directors, coroners, registrars to cover:
Death certification; process and errors, production of summary statistics; organisational issues.

Results: Death certification with the production of summary statistics might be seen as a sine qua non of a civilised society. Nevertheless currently in the UK, the process of death certification, registration and exclusion of unnatural causes of death is labyrinthine, with multiple conflicting responsibilities and lines of accountability. Descriptions of roles date back hundreds, if not thousands of years. Previous reports have made a number of recommendations, although current legislation has not been changed since 1926. An organogram showing the multiple conflicting lines of responsibility and accountability suggests that the process is long overdue for reform. Doctors are highly critical of the (separate) certification procedures for authorising cremation - although this accounts for the disposal of $75 \%$ of bodies. A new role - that of a medical examiner has been suggested by a number of stakeholders as rationalising the process and ensuring that the three functions of death certification - legal, epidemiological and practical (for disposal of the body) are improved. However there are mixed views about the introduction of this new tier.

Conclusions: Death certification practice in the UK is long overdue for reform. In particular the organisation of the coronial system needs to be updated. Although there are mixed views about medical examiners - there is an important co-ordinating role which needs fulfilling.

\section{HOSPITAL MORTALITY ASSOCIATED WITH DAY AND TIME OF ADMISSION TO INTENSIVE CARE UNITS IN ENGLAND, WALES AND NORTHERN IRELAND}

H. Wunsch', J. Mapstone', R. Hanks², T. Brady', K. Rowan'2. 'Department of Epidemiology and Population Health, London School of Hygiene \& Tropical Medicine, Keppel Street, London; ${ }^{2}$ Intensive Care National Audit \& Research Centre (ICNARC), Tavistock House, Tavistock Square, London

Background: Intensive care units (ICUs) are set up to deliver constant care 24 hours a day, 7 days a week. However, the first few hours after admission to an ICU are often vital for commencing and establishing treatment plans for patients. Therefore we investigated whether outcome was associated with the day of the week or time of admission to intensive care.

Methods: Day of the week was defined as the period from 08:00 to $07: 59$ hours the following day. Time of day was defined as "day" $08: 00$ to $17: 59$ hours, "evening" 18:00 to $23: 59$ hours and "night" 00:00 to 07:59 hours. These definitions were applied to 75,621 admissions to 102 ICUs from December 1995 to February 2000 in the UK Case Mix Programme Database. After exclusion of re-admissions to ICU within the same hospital stay, and those ineligible for the APACHE II case-mix adjustment calculation, 56,049 admissions were analysed. Crude and case-mix adjusted hospital mortality were examined by day of the week and time of admission to the ICU.

Results: Patients admitted on Saturday and Sunday had a higher hospital mortality compared with admissions on Wednesday (Saturday crude odds ratio (OR) 1.47, 95\% Cl 1.37-1.58; Sunday OR 1.49, 1.38-1.60). The association was still significant after adjustment for probability of death using the UK APACHE II equation (Sat OR 1.20, 1.11-1.30; Sun OR 1.17, 1.08-1.27). However, after further adjustment using either the individual components of the APACHE II or APACHE III model the differences were no longer significant. Night admissions were also associated with higher mortality compared with day (crude OR 1.44, 1.37-1.51) and after UK APACHE II adjustment for case-mix (OR 1.16, 1.10-1.23). Again, further adjustment for case-mix using components of the APACHE II and APACHE III models reduced the difference in mortality to a nonsignificant level.

Conclusions: After appropriate adjustment for case mix, the day of the week and time of day of admission of patients to the ICU were not significantly associated with hospital mortality. The frequently used technique of risk adjustment with the current UK APACHE II equation did not adequately capture the differences in case mix. Further assessment of risk adjustment models is needed for future critical care research.

\section{DEPRIVATION AND RISK OF UNPLANNED EMERGENCY MEDICAL READMISSION. WHAT THE PERFORMANCE INDICATORS WILL NOT TELL}

G. Liratsopulos', D. Havely', G. Cook'. 'Department of Epidemiology, Stepping Hill Hospital, Stockport NHS Trust, Stockport

Objective: To examine the effect of deprivation on the risk of emergency medical readmission (EMA). 
Design: Multivariate proportional hazards model, based on routine data (HES). Socioeconomic status (SES) was measured ecologically. Five deprivation groups were defined using patients' post codes and the DETR Index of Multiple Deprivation Score for electoral wards.

Setting and study period: A District General Hospital in Greater Manchester, 4.5-year period 1997-2001.

Participants: All EMA spells during the study period leading to a live discharge at the end of the index EMA were included in the study $(n=33,515)$.

Main outcome measures: Hazard ratio (HR) of unplanned EMA by patient's deprivation group, adjusting for sex, age group, admission method, select group of diagnoses and length of stay (LoS) as a surrogate for disease severity.

Results: With the least deprived group as reference and the most deprived group defined as group 5, the HR of unplanned readmission was $1.16\left(p:<0.001^{* * *}\right), 1.25\left(p:<0.001^{* * *}\right), 1.4$ $\left(p:<0.001^{* *}\right)$ and $1.6\left(p:<0.001^{* * *}\right)$ for deprivation groups $2-5$ respectively.

Women had a lower readmission risk (HR: 0.92, p: $<0.001^{* * *}$ ) and so did patients who were referred by a GP (HR:0.85, p: $<0.001^{* * *}$ ) and patients with a CVA (HR: 0.7, p:<0.001***). Patients aged $60-74$ and $>75$ had higher readmission risk (HR: 1.36 and 1.36 respectively, $\left.\mathrm{p}:<0.001^{* *}\right)$ and so did patients with heart failure (HR: 1.46, $\mathrm{p}:<0.001^{* * *}$ ), acute coronary syndromes/chest pain (HR: 1.17, $p_{:}<0.001^{* * *}$ ) and COPD/asthma (HR: 1.51, $\left.\mathrm{p}:<0.001^{* * *}\right)$. With the lowest LoS quartile as reference, the readmission HR was $1.31\left(p:<0.001^{* * *}\right), 1.55\left(p:<0.001^{* * *}\right)$ and $1.69\left(p:<0.001^{* * *}\right)$ for quartiles $2-4$ respectively.

Conclusions: Deprivation exercises an independent effect on the risk of readmission after an EMA. Readmissions can be preventable (e.g. due to sub-optimal healthcare) or non-preventable (e.g. due to non-modifiable disease progression). Estimates of the proportion of readmissions that are due to preventable healthcare factors vary between 9 and $48 \%$. It is unlikely that deprivation status has an effect on the quality of emergency care. The results therefore support that lower SES is either associated with increased disease severity or other factors such as poor patient support system in the community. Re-admissions are included in the DoH performance indicators both for NHS trusts and PCTs and differences in "deprivation mix" can influence how well a hospital or PCT performs. We would support that the indicators are adjusted for patients' SES in the future, if NHS organisations serving deprived communities are not to be unfairly judged.

\section{ASSESSMENT OF LONG-TERM PUBLIC HEALTH BENEFITS FROM SHORT-TERM INTERVENTIONS: OBESITY AS A CASE STUDY}

L.S. Aucott', A.S. Poobalan', W.C.S. Smith' . 'Department of Public Health, University of Aberdeen, Aberdeen

Background: Health intervention policies are usually based on randomised controlled trials (RCT's) with relatively short follow-up. However, some health issues require longer-term assessment of the intervention impact, and RCT results would only be indicative and may even mislead. One such case is obesity, now a recognised chronic disease, described as an escalating epidemic by the WHO. It is associated with a wide variety of co-morbidities causing premature death or debilitating illness. Many short-term studies demonstrate that weight loss can ameliorate and in some cases eliminate these disorders. However, do these benefits continue? The long-term evidence of weight loss impact seems to be limited.

Objectives: To assess the long-term health outcomes after weight loss for the obese.

Methods: A systematic search of literature published between 1966 to 2001 was under taken on four bibliographic databases. Studies were limited to obese people $\left(B M l \geqslant 28 \mathrm{kgs} / \mathrm{m}^{2}\right)$, with preferably 5 years follow-up, weight loss measurements and at least one other health outcome recorded. The long-term impacts of short-term changes were modelled.

Results: A total of 7567 abstracts were identified. 302 were selected for critical appraisal. After application of the inclusion criteria 37 studies were reviewed. These provided some evidence of long-term benefits of weight loss on health outcomes. The effects of weight loss with respect to mortality were significantly beneficial for women, with obesity related illness, who intentionally lost weight. Intervention for obesity also appears to reduce the risk of developing diabetes and indeed improve diabetes status. By modelling over a range of weight differences an average loss of $10 \mathrm{kgs}$ produced an expected drop of $0.28 \mathrm{mmol} / \mathrm{L}$ in cholesterol levels and a $3 \mathrm{mmHg}$ drop in diastolic blood pressure for obese people. However, the lack of information about the occurrence of weight loss between baseline and final follow-up meant that assessing whether weight losses were maintained, cycled or regained was difficult. Most were cohort/ prospective studies which although long-term often had poor percentage follow-up of patients. Some studies assessed weight loss retrospectively by questionnaires.

Conclusions: Weight loss providing health benefits have been indicated from this review. However, the quality of reporting and/or methodological flaws of the studies prevent further endorsement. Short-term studies show evidence of health benefits, however, modelling based on epidemiological studies is important to assess the longterm public health benefits of these interventions. This approach is relevant to other short-term interventions for chronic illness.

\section{Obesity and diabetes}

\section{TRENDS IN BMI OF YOUNG ADULTS: RESULTS FROM GLASGOW UNIVERSITY STUDENTS BORN 1918 TO 1951}

M. Okasha', G. Davey Smith', P. McCarron'2, J. McEwen ${ }^{3}$ ' 'Department of Social Medicine, University of Bristol; ${ }^{2}$ Department of Epidemiology \& Public Health, N. Ireland Cancer Registry, The Queen's University of Belfast; ${ }^{3}$ Department of Public Health, University of Glasgow

Objectives: To determine whether the recent trends in obesity were occurring in the earlier part of the $20^{\text {th }}$ Century.

Design: Cross-sectional analysis of data that were collected over a 20 year period.

Setting: Student Health Service of the University of Glasgow, 1948 to 1968 .

Participants: 9,199 men and 2,528 women, born between 1918 and 1951, who had their height and weight measured while at university.

Main outcome measures: Mean Body Mass Index (BMI) and proportion of people overweight $\left(B M l>=25 \mathrm{~kg} / \mathrm{m}^{2}\right)$ per year of birth.

Results: The mean age of the students at the time of measurement was 20.6 years in men and 19.3 years in women. In crude analyses, the mean BMI did not change over time. However, the age of the students, particularly the males, decreased over time, possibly because those attending in the earlier years were undergoing compulsory military service. In age-adjusted models, there was clear evidence of a higher BMI among later born cohorts of men. The mean BMI in the earliest birth cohorts quintile (1918 to 1930) was $21.1 \mathrm{~kg} / \mathrm{m}^{2}$ (standard error (SE): $0.05 \mathrm{~kg} / \mathrm{m}^{2}$ ), compared to $21.9 \mathrm{~kg} / \mathrm{m}^{2}$ (SE: 0.06 $\mathrm{kg} / \mathrm{m} 2$ ) in latest quintile (1946 to 1951). The corresponding values for women were $21.3 \mathrm{~kg} / \mathrm{m}^{2}$ (SE: $0.15 \mathrm{~kg} / \mathrm{m}^{2}$ ) and $21.3 \mathrm{~kg} / \mathrm{m}^{2}$ (SE: $0.10 \mathrm{~kg} / \mathrm{m}^{2}$ ) respectively. Linear regression models indicated that each one year later a man was born, his BMI was on average 0.04 $\mathrm{kg} / \mathrm{m}^{2}$ (95\% Cl: 0.03 to 0.05$)$ higher. This association was not altered by adjusting for childhood socio-economic position or smoking behaviour while at university. For women, there was no evidence of a linear relationship ( $\beta$ coefficient: $0.00 \mathrm{~kg} / \mathrm{m}^{2}, 95 \% \mathrm{Cl}:-0.02$ to 0.01 ). Similar patterns were seen when the proportion of individuals who were overweight was used as an outcome. The age-adjusted odds ratio of being overweight in the latest compared to the earliest birth cohort quintile was 2.91 (95\% Cl: 2.15 to 3.95$)$ in men and $0.87(95 \% \mathrm{Cl}$ : 0.49 to 1.56 ) in women.

Conclusions: The trends of increasing BMI in young people which have been observed in recent decades were also occurring in the early part of the $20^{\text {th }}$ century, at least in males. The environmental changes underlying these trends are not exclusively a recent phenomenon and were already operating around the mid- $20^{\text {th }}$ century.

\section{OBESITY, ETHNICITY AND SOCIOECONOMIC STATUS IN EAST LONDON SCHOOL CHILDREN: FINDINGS FROM THE RESEARCH IN EAST LONDON ADOLESCENTS COMMUNITY HEALTH STUDY (RELACHS)}

S.J.C. Taylor', R. Viner'2, J. Head',2, H. Tate', R. Booy', M. Haines', S. Stansfeld', K. Bhui', S. Hillier'. 'Institute of Community Health Sciences, Queen Mary, University of London; ${ }^{2}$ University College, London

Aims: To examine the prevalence of obesity, overweight, self-reported diet and physical activity by sex, ethnicity and socioeconomic status in a deprived multi-ethnic population in East London.

Methods: School based, cross-sectional survey of a representative sample of 2790 children from Year 7 (11-12 years) and Year 9 
(13-14 years) in East London in 2001. Obesity was defined as body mass index (BMI, weight/height $\left.{ }^{2}\right)>95^{\text {th }}$ centile (UK 1990 growth reference), overweight as BMI between $85^{\text {th }}$ and $95^{\text {th }}$ centile. Socioeconomic status (SES) was examined using eligibility for free school meals, access to a car, parental unemployment, 'crowding index' (people per room at home), and postcode.

Results: 2790 children participated (response rate $84 \%$ ), 49\% male. BMI was available for $90 \%$ of whom $27 \%$ were "white", $25 \%$ Bangladeshi, 21\% "black", 9\% Indian and 7\% Pakistani. 91\% had lived in the UK for at least five years. Overall $20 \%$ boys $195 \% \mathrm{Cl} 18 \%$, $22 \%)$ and $22 \%$ girls $(19 \%, 25 \%)$ were obese and a further $16 \%$ boys $(14 \%, 19 \%)$ and $14 \%$ girls $(12 \%, 16 \%)$ were overweight. The groups with the highest proportions of obese or overweight children were the Indian males $(47 \%, 39 \%, 55 \%)$ and "black" females $(42 \%, 36 \%$, $48 \%)$. Similar proportions of white males $(22 \%)$ and Bangladeshi males $(20 \%)$ were obese. Fewer Bangladeshi females (14\%) were obese compared to white females $(25 \%)$. $31 \%$ of the boys and $25 \%$ of the girls reported eating at least three high fat snacks or meals per day, Bengali children ate more high fat foods than the other ethnic groups. Overall $43 \%$ of girls and $23 \%$ of boys reported taking little or no exercise outside school hours and, within sex, there was little difference in the frequency of exercise reported between the ethnic groups. There were no significant associations between the measures of SES and obesity.

Conclusions: Overall around one third of the children surveyed were obese or overweight, a third reported taking very little or no physical exercise and a quarter reported eating at least three high fat snacks/meals each day. Socioeconomic status, measured in a variety of ways, was not related to obesity in this relatively deprived, multi-ethnic, adolescent population. An 'epidemic' of obesity has been described amongst British children - these data suggest that girls and/or boys from some ethnic groups may be at particular risk.

\section{PREVALENCE OF DIAGNOSED DIABETES MELLITUS IN GENERAL PRACTICE IN ENGLAND AND WALES, 1994 TO 1998}

R. Ryan', A. Newnham', K. Khunti' ${ }^{2}$, A. Majeed ${ }^{3}$. 'Office for National Statistics, London; ${ }^{2}$ Department of General Practice and Primary Health Care, University of Leicester; ${ }^{3}$ School of Public Policy, University College London

Objectives: To estimate the prevalence of diagnosed diabetes mellitus in general practice in England \& Wales from 1994 to 1998. To describe variations in prevalence and treatment type by age, sex, deprivation category and year. To estimate future numbers of people with diagnosed diabetes.

Design: Analysis of electronic patient records from the General Practice Research Database.

Setting: 210 general practices in England \& Wales.

Main outcome measures: Age-specific and age-standardised prevalence of diabetes in each analysis year and in each deprivation quintile. Proportions of diabetic patients who were diet controlled, oral hypoglycaemic controlled and insulin treated. Projected numbers of people with diagnosed diabetes in 2023 based on prevalence in 1998.

Results: The age-standardised* prevalence of diagnosed diabetes increased from 1.89 to 2.23 per 100 males, and from 1.37 to 1.64 per 100 females from 1994 to 1998 . Prevalence increased in most age groups, was higher in males than females in most age groups and peaked in those aged $75-84$ years in each year. Overall, $29 \%, 48 \%$ and $24 \%$ of males, and $26 \%, 48 \%$ and $26 \%$ of females respectively were diet controlled, oral hypoglycaemic controlled and insulin treated. The prevalence of diabetes increased with increasing deprivation in men and women aged 35-54 years but this gradient was not consistent in younger and older age groups. If prevalence increases by $10 \%$, the number of people with diagnosed diabetes is projected to rise from 1.15 million in 1998 to 1.66 million in 2023 .

Conclusions: The observed increase in prevalence from 1994 to 1998 was probably due to increased survival, case ascertainment and incidence. Because the population is ageing, the number of people with diagnosed diabetes is projected to rise even if there is no further increase in age-specific prevalence. Even with a conservative increase in prevalence of $10 \%$ from 1998 to 2023, the number of people with diabetes is projected to rise by $44 \%$. tion.

\section{TESTING THE VALIDITY OF THE FRAMINGHAM EQUATION IN TYPE 2 DIABETES: A UK COMMUNITY-BASED 4-YEAR PROSPECTIVE STUDY}

R. Mehta', M. Mullee', R. Guzder', W. Gatling'. 'Health Care Research Unit, University of Southampton; ${ }^{2}$ Department of Diabetes, Poole Hospital NHS Trust, Poole

Objective: Charts and computer programs based on the Framingham equation are in widespread use. We have investigated the accuracy of the Framingham equation in predicting the onset of primary cardiovascular disease (CVD) in newly diagnosed Type 2 diabetes.

Design: The study was carried out in a defined community based cohort of 738 patients with a new diagnosis of Type 2 diabetes made between $01 / 05 / 96$ and 30/06/98.

Setting: A community in Poole, England.

Participants: Four hundred and twenty eight patients aged 30-74 (Mean age 59 (SD \pm 0.5$), 245$ male and 183 female) and free of CVD were entered into the analysis.

Main outcome measures: Framingham risk scores for CVD were calculated for the period of observation.

Results: Median follow-up was 4.2 years $(S D \pm 0.62)$ and cardiovascular disease status was ascertained at the time of review or from primary care notes and mortality data. $95 \%$ confidence intervals were calculated both for predicted and actual events. Based on the Framingham Equation, the predicted number of events was 64 $(95 \% \mathrm{Cl} 60$ to 67$)$ and the actual number of primary cardiovascular events seen was $98(95 \% \mathrm{Cl} 77-111)(\mathrm{p}<0.001)$. The difference was sustained both at the lowest and highest tertiles of predicted risk.

Conclusions: In our population, the Framingham equation underestimates cardiovascular risk by at least a third. Current guidelines for intervention based on Framingham-derived risk scores may leave a substantial number of individuals with Type 2 diabetes under-treated.

\section{Pregnancy and parenting}

\section{RED CELL FOLATE LEVELS IN NON-PREGNANT WOMEN AGED 20-34 YEARS IN SOUTHAMPTON}

H.M. Inskip, S.E. Borland, R.A. Bayly, J.F. Hammond, S.M. Robinson, K.M. Godfrey. MRC Environmental Epidemiology Unit, University of Southampton, Southampton General Hospital, Southampton, SO 16 6YD

Objectives: To examine the influences on red cell folate concentration (RCF) in non-pregnant women of childbearing age.

Design: The data were collected as part of the Southampton Women's Survey, a prospective study of non-pregnant women of childbearing age. The interview comprised details about health and lifestyle, an administered food-frequency questionnaire and information about nutritional supplement intake. Venous blood samples were taken and RCF was assessed by microparticle enzyme immunoassay.

Setting: Southampton, UK.

Participants: 3820 women aged 20-34 resident in Southampton, interviewed between April 1998 and June 2000, who provided a blood sample. The participants were representative of the British population in terms of ethnicity, and had a similar range of Townsend index scores (a measure of area deprivation).

Main outcome measures: Red cell folate concentration in $\mathrm{nmol} / \mathrm{l}$. The distribution was skewed so a logarithmic transformation was used. Multiple regression analysis was used to assess the percentage effect on RCF of a unit change in each independent variable.

Results: The median RCF was $693 \mathrm{nmol} / /$ (inter-quartile range $535-918)$. Some 82 women were taking $400 \mu g /$ day or more of folic acid - the amount recommended for those trying to get pregnant. In the regression model, use of supplements containing folic acid was an important influence on RCF, as $400 \mu \mathrm{g} / \mathrm{d}$ increased RCF by $36 \%$ $(95 \% \mathrm{Cl} 30 \%-42 \%)$. Of the dietary factors considered, breakfast cere-
als were the most influential; each additional daily bowl of cereal increased RCF by $19 \%(95 \% \mathrm{Cl} 16 \%-22 \%)$. Consumption of five or more portions of fruit and vegetables each day increased RCF by $11 \%$ $(95 \% \mathrm{Cl} 7 \%-15 \%)$ compared with those consuming fewer than two portions. RCF was $8 \%(95 \% \mathrm{Cl} 6 \%-10 \%)$ lower in those who smoked, and $13 \%(95 \% \mathrm{Cl} 8 \%-18 \%)$ lower in those without qualifications compared to those with a degree, and $4 \%(95 \% \mathrm{Cl} 1 \%-7 \%)$ lower in those in receipt of benefits. Social class, Townsend index score, and body mass index did not influence RCF, after adjustment for the above factors. The influences identified were similar in those who did and did not take any supplements containing folic acid. 
Conclusion: Measures that lead to increased intakes of breakfast cereals, fruit and vegetables and that limit cigarette smoking are likely to improve the folate status of young women. Such measures may be of particular importance to those with lower levels of education or who are receiving benefits.

\section{INEQUALITIES IN MATERNAL MORTALITY IN ENGLAND AND WALES DURING THE TWENTIETH CENTURY: WHAT LIES BEHIND THE CHANGING GRADIENTS?}

A. Macfarlane. Department of Midwifery, St Bartholomew School of Nursing and Midwifery, City University

Background: The most recent Confidential Enquiry into Maternal deaths, covering the years 1997-99, found strong social class differences in maternal mortality. This contrasts both with the inverse social class gradients observed in the early 1930s and historical reviews suggesting that social factors did not play a major role in maternal mortality in the first half of the twentieth century.

Objective: To document changing inequalities in maternal mortality during the twentieth century and assess the impact of other changes over the same period.

Setting: England and Wales, 1915-1984, United Kingdom, 1985-99.

Design and sources: Retrospective review of Registrar Generals' decennial supplements, reports of confidential enquiries into maternal deaths and other analyses of death statistics.

Findings: In 1930-32, mortality rates per 1,000 'legitimate' live births were highest in social class II and lowest in social class $V$ for several categories of death. Paradoxically, when expressed per 1,000 population, maternal mortality rates were highest among women with husbands in manual occupations. Data from decennial supplements for the 1950s to the 1980s found no overall inverse social class gradient when births were used as a denominator, but a tendency for raised maternal mortality rates among women with partners in social classes I and II. Social class differences in maternal mortality returned to the public agenda in 2001, when marked gradients in maternal mortality were found in the Confidential Enquiry for 1997. 99, but these rates were constructed on a different basis than those for earlier years, making use of both the woman's own social class and her partner's.

Discussion and conclusions: Analyses of social class differences in maternal deaths published from the 1930s onwards showed changing gradients, but the categories of deaths included, the ways in which they were classified, the degree of ascertainment of pregnancy-related deaths, the women included in the analyses, the extent to which they were classified by their own or their partner's occupation and the denominators used also changed. Over the same period, the overall maternal mortality rate fell markedly from the mid 1930s onwards and there were major changes in the nature and accessibility of maternity care.

It is unclear to what extent methods of measurement and ascertainment have contributed to changes in inequalities in maternal mortality, but with the decline in direct obstetric deaths and better ascertainment of indirect deaths, the social factors behind pregnancy-related death are clearly back on the public health agenda.

\section{PARENTING AND PUBLIC HEALTH: SYSTEMATIC REVIEW OF COHORT STUDIES INVESTIGATING THE LINK BETWEEN RELATIONSHIPS IN THE HOME DURING CHILDHOOD AND PHYSICAL HEALTH AND DISEASE IN LATER LIFE}

S. Stewart-Brown, R. Shaw, L. Morgan. Health Service Research Unit, University of Oxford, Institute of Health Sciences, Old Road, Headington, Oxford OX3 $7 L F$

Background: The quality of relationships between adults has been shown to play a part in determining susceptibility to disease, particularly heart disease and mortality in general. The quality of relationships in the home might therefore play a part in determining child health. Relationships with parents in childhood influence emotional and social development and may thus influence the quality of relationships children make with others both in childhood and adulthood. This review aimed to test the hypothesis that, through these links, the quality of the parent- child relationship could be a determinant of health and disease in later life.

Methods: Systematic search of Medline, Sociological Abstracts and Psych Info for studies which measured any aspect of the quality of relationships in the home during childhood and any aspect of physical health or disease in the child at a later point in time. Application of pre-determined inclusion criteria. Data extraction of all included studies. Critical appraisal according to standard criteria for assessing validity of longitudinal studies. Qualitative synthesis of results.

Results: 11914 titles and abstracts were downloaded and 628 papers obtained. 44 relevant papers were identified covering 36 studies in 34 cohorts. Studies ranged from $39-5000$ subjects in size and six months to 35 years in length. They were classified into groups according to the health outcomes measured $(2$ studies provided results for two groups of outcomes). Seven studies reported general health/ symptoms of illness, four disease outcomes, six injuries, one asthma, two haemodynamic stress, five some aspect of growth, height or weight, six timing of puberty and seven teenage pregnancy. Most studies focused on conflict, hostility and criticism, but other aspects of parenting were covered. Results were classified as positive, partially positive, no relationship or negative as follows: positive:22; partially positive: 8 ; no association: 10 ; negative association: 1 . The quality of studies varied, however there was no discernible relationship between study quality and results.

Conclusions: Publication bias may have influenced the results. However, included studies lend support to the hypothesis that the quality of relationships in the home during childhood is a determinant of health and susceptibility to disease both in childhood and in later life. Further studies are needed to establish the extent to which the relationship is causal and to clarify which aspects of parenting influence which aspects of health.

\section{THE PARENTAL PROCESS OF MAKING DECISIONS TO HELP THEIR OVERWEIGHT CHILD}

L.D. Edmunds. Department of Public Health, University of Oxford, Oxford

Objective: to elucidate the process parents' go through when making decisions about how to help their overweight child and to identify factors that determine action (or non-action).

Design: Semi-structured interviews with body shapes used as prompts. Analysis was thematic and iterative.

Setting: Two geographical areas of the United Kingdom.

Participants: 40 parents of school-age children of (4-15 years) with concerns about their children's weight.

Results: A general sequence of stages in the process was identified. These were apparent regardless of the child's age or sex. Parents were aware that their child was larger than their peers and monitored their growth. Children came home from school saying they were being teased about weight at moderate levels of adiposity. Parents still hoped that their child might grow out of it but if the child remained overweight with growth, they made judgements based on other family members' weight status. Actions to change diet and physical activities were initially covert. Many had been to weight loss groups and were applying this knowledge at the family level to manage their child's weight. All were aware of the importance of keeping them active, but this was often problematic in practice. They were also concerned about precipitating eating disorders. As weight became more important to the child with rising awareness, usually due to bullying, manipulation of diet and activity within the family became overt. However, sensitivity and non-medicalisation remained paramount. Overweight was perceived as a health problem at a specific body shape that approximately equates to the $85^{\text {th }} \mathrm{BMI}$ centile. This shape was consistently selected regardless of a child's sex or age. Medical advice was sought at this shape, often triggered by bullying. Parents' experiences in relation to their child's weight with health professionals and school staff were varied.

Conclusions: Parents seek professional help when the child's overweight is well established. An earlier period is apparent when parents are seeking help but do not want their child labelled medically. The opportunity may exist to intervene at an earlier stage if the right approach can be devised.

\section{Coronary heart disease and stroke}

\section{CORONARY HEART DISEASE AMONG DIABETIC AND NON-DIABETIC PEOPLE: SOCIO-ECONOMIC AND GENDER DIFFERENCES IN INCIDENCE, PROGNOSIS AND MORTALITY}

E. Forssas', I. Keskimäki', A. Reunanen², S. Koskinen². 'National Research and Development Centre for Welfare and Health (STAKES), Helsinki; ${ }^{2}$ Department of Health and Disability, National Public Health Institute, Helsinki

Background: Coronary heart disease (CHD) is a major complication of diabetes mellitus. The data on this association are mainly based on 
mortality studies, but the incidence and prognosis of non-fatal events have seldom been explored. The study investigates CHD morbidity and mortality and their patterning by socio-economic status and gender.

Methods: The baseline cohort of diabetic people drawn from the 1991-1996 Social Insurance Reimbursement Register comprised all Finns aged 35-74 entitled to free anti-diabetic medicines. Two age and sex matched non-diabetic referents were chosen from the same register. Outcome events in the maximum follow-up of 6 years were identified in the Social Insurance Register on chronic diseases, and National Hospital Discharge and Causes of Death Registers using personal identification codes. Data on present or pre-retirement occupation, education and disposable family income came from the 1970-1990 population censuses.

Results: The age-standardised CHD mortality among diabetic person was for women 604 and for men 1203 per $10^{5}$ person years corresponding to the relative mortality rates of 6.04 (5.62-6.50) in women and 3.42 (3.25-3.59) in men compared to non-diabetic referents. Relative differences for CHD incidence and survival after CHD diagnosis between diabetic and non-diabetic people were somewhat smaller with the incidence and mortality ratios of 3.55 (3.43-3.67) and $2.99(2.65-3.36)$ in women, and $2.64(2.56-2.72)$ and 2.05 (1.88-2.24) in men. Gender and socio-economic differences in CHD incidence and mortality were generally larger among non-diabetic than diabetic people. Crude Kaplan-Meier estimates for five year mortality after CHD diagnosis were $28.1 \%$ among diabetic women and $30.4 \%$ among diabetic men but $9.9 \%$ and $16.3 \%$ in non-diabetic women and men. Socio-economic differences in prognosis were small, except for the lowest income quintile, where the hazard ratio among diabetic people was $1.50(1.12-2.01)$ for women and 1.38 (1.14-1.66) for men compared to the highest income quintile. Corresponding hazard ratios among non-diabetic referents were equally large; 1.43 (0.86-2.39) for women and 1.53 (1.18-1.99) for men.

Conclusions: Higher incidence and poorer prognosis are both important contributors to excess CHD mortality among diabetic persons. Gender differences in CHD mortality are smaller in diabetic than non-diabetic persons which results from reduced gender differences in CHD incidence and prognosis among diabetic people. Socio-economic differences in mortality from CHD among both diabetic and non-diabetic persons are mainly due to differential incidence.

\section{EXPLAINING THE DECLINE IN CORONARY HEART DISEASE MORTALITY IN ENGLAND AND WALES, 1981 -2000}

B. Unal Aslan, J. Critchley, S. Capewell. Department of Public Health, University of Liverpool

Introduction: The UK death rate from coronary heart disease (CHD) is still very high. However, like most developed countries, UK mortality rates from (CHD) have been falling since the 1970s.

These falls need to be explained to better predict future trends and explore different policy options for CHD prevention.

Objective: To explore how much of the fall in coronary heart disease (CHD) mortality in England and Wales between 1981 to 2000 can be attributed to "evidence based" medical and surgical treatments, and how much to changes in major cardiovascular risk factors.

Methods: The cell-based IMPACT mortality model previously validated in Scotland and New Zealand; was further developed and refined to combine data for England and Wales describing: a) effectiveness of specific cardiological treatments and risk factor reductions, b) CHD patient numbers, c) uptake of specific medical and surgical treatments and d) population trends in major cardiovascular risk factors (smoking, cholesterol, hypertension, obesity, diabetes, physical activity and deprivation).

The main sources were ONS official statistics \& BHF Annual CHD Statistics, (for population and patient data), the British Regional Heart Study, General Household Survey, \& Health Survey for England (risk factor data), various national and local clinical audits and surveys (treatment prescription \& uptake) and published RCTs, meta-analyses and population studies (efficacy of interventions). The regression coefficients in the existing model (quantifying the fall in CHD mortality attributable to the change in a specific risk factor) were reviewed, and updated.

Results: Between 1981 and 2000, CHD mortality rates fell by almost $40 \%$ in England and Wales, with 80,417 fewer CHD deaths than expected from baseline mortality rates in 1981 . Some $47 \%$ of this fall was attributed to treatments in individuals [acute myocardial infarction $9 \%$; secondary prevention $8 \%$, heart failure $14 \%$, angina $9 \%$, and hypertension $7 \%]$, and $53 \%$ to population risk factor reductions [smoking $28 \%$, cholesterol $13 \%$, blood pressure $9 \%$ and $3 \%$ from beneficial changes in other factors, which could include Barker and deprivation]. Adverse trends were seen for obesity, physical activity, and diabetes.

The proportional contributions remained relatively consistent using a robust sensitivity analysis.

Conclusions: Approximately half the coronary heart disease mortality fall in England and Wales 1981-2000 was attributed to medical therapies, and half to reductions in major risk factors. These findings emphasise the importance of a comprehensive CHD strategy, which maximises the population coverage of effective treatments and secondary prevention programmes, and actively promotes primary prevention, particularly for diet, and smoking.

\section{REDUCING CORONARY HEART DISEASE MORTALITY IN ENGLAND AND WALES BY INCREASING THE UPTAKE OF CARDIOLOGICAL TREATMENTS}

B. Unal Aslan', J. Critchley', J. McMurray ${ }^{2}$, S. Capewell'. 'Department of Public Health, University of Liverpool; ${ }^{2}$ The Clinical Research Initiative in Heart Failure, University of Glasgow

Introduction: Coronary heart disease (CHD) is the largest cause of mortality in the United Kingdom, accounting for some 1 10,000 deaths annually with half occurring prematurely. A large number of evidencebased cardiological interventions are effective in reducing coronary deaths. However, recent clinical audits and studies suggest that many eligible patients fail to receive appropriate therapies. These low treatment uptake rates result in avoidable morbidity and mortality.

Objective: To determine the extent to which increases in the uptake of effective cardiological treatments could further reduce CHD mortality in England and Wales.

Methods: We used the cell-based IMPACT mortality model, previously validated in Scotland and New Zealand, for the England and Wales population of 51 million. We combined data on: a) CHD patient numbers, b) uptake of specific medical and surgical treatments, c) effectiveness of specific cardiological treatments and risk factor reductions, and d) population trends in major cardiovascular risk factors.

We then determined the magnitude of $\mathrm{CHD}$ mortality reduction potentially achievable through increased uptake of specific treatments in the year 2000 .

Results: In 2000, approximately 38,083 deaths (minimum estimate 29,753 , maximum estimate 50,168 ), were prevented or postponed for at least one year by medical and surgical CHD treatments. However, uptakes were generally poor; for instance, approximately $30 \%$ for aspirin treatment of community angina and $40 \%$ for thrombolysis for acute myocardial infarction.

Increasing treatment uptake to $100 \%$ of all eligible patients was considered unrealistic. However, increasing uptake up to $80 \%$ (the NSF CHD target) would have prevented or postponed a further 35,720 deaths $144 \%$ from increases in secondary prevention therapies, and $14 \%, 12 \%, 10 \%$, and $7 \%$ from increasing the treatments for heart failure, community angina, acute myocardial infarction and hypertension respectively). In contrast, potential gains from doubling the current provision of CABG surgery $(26,311$ in 2000) would have been modest.

Conclusions: Many eligible patients are currently not receiving appropriate and effective cardiological treatments. This is leading to eminently avoidable disease and death. Increasing the uptake of CHD treatments could have prevented or postponed approximately 35,000 deaths in England \& Wales in 2000, almost doubling the benefit actually achieved from therapy. Comparable symptomatic benefits might also be expected.Future strategies and clinical audits should therefore prioritise the delivery of secondary prevention therapies to all eligible CHD patients.

\section{SECULAR TRENDS IN MORTALITY BY STROKE SUBTYPE OVER THE TWENTIETH CENTURY: RESOLUTION OF THE STROKE-CORONARY HEART DISEASE PARADOX?}

S. Ebrahim', D.A. Lawlor', G. Davey Smith', D.A. Leon², J. Sterne' 'Department of Social Medicine, University of Bristol; '2Department of Epidemiology, London School of Hygiene \& Tropical Medicine

Background: A paradox regarding the epidemiology of stroke is that despite sharing many risk factors with coronary heart disease there was no twentieth century epidemic of stroke to match that seen for coronary heart disease. Analysis of secular trends in stroke subtypes may resolve this paradox, but routine mortality data do not accurately distinguish between stroke subtypes. The aim of this study was to estimate 
secular trends in cerebral infarct and cerebral haemorrhage over the last century for England and Wales to see if the trends in cerebral infarct and coronary heart disease were similar, as one would expect from our understanding of their similar pathology.

Methods: The ratio of cerebral infarct to cerebral haemorrhage was calculated from all available sources, over the last century, of autopsy or brain imaging studies. These sources were identified from a Medline search (1966-September 2001), bibliographic searches and contact with experts in the field. The ratios obtained from these sources were modelled to estimate the ratio of cerebral infarct to cerebral haemorrhage for every year from 1932 to 1999 (range of years for which adequate data were available). These estimated ratios were used to derive rates of cerebral infarct and cerebral haemorrhage from the total stroke mortality rate, obtained from the Office for National Statistics, for each year.

Findings: The ratio of cerebral infarct to cerebral haemorrhage increased from 0.5 in 1932 to 5.0 by 1999. Estimated secular trends by stroke subtype suggested that whilst there was a consistent decline in mortality from cerebral haemorrhage over the last century, mortality from cerebral infarct increased to a peak in the early 1970 s and then declined. Trends in estimated cerebral infarct mortality closely matched those for coronary heart disease mortality.

Implications: These results provide evidence of important differences in aetiology between cerebral infarct and cerebral haemorrhage. The aetiology of cerebral infarct appears closely related to that of coronary heart disease, but cerebral haemorrhage aetiology must differ importantly from these.

\section{Mental health I}

\section{MENTAL HEALTH PROBLEMS AT WORK: A MAJOR CAUSE OF CONCERN}

F. Ford, J. Gorry, A. Jacoby. Department of Primary Care, University of Liverpool, $L 693 G B$

Background: Mental health problems (mainly depression) are now the most common cause of certified work incapacity, particularly of over 3 months' duration, and are more common in women and in the south of England. Much research has been done around the stigma attached to mental illness and other conditions such as epilepsy, which may lead to problems in job recruitment and retention. This study aimed to test the attitudes of people to co-workers with a variety of different medical problems.

Method: A question was devised for the November 2001 National Statistics Omnibus Survey, which asked subjects in England, Scotland and Wales to rank in order of which would cause the most concern, a new work colleague who: 1) was over 50, 2) was a wheelchair user, 3) was epileptic and had 2 seizures in the last year, 4) had a heart attack a year ago, 5) had been off sick with stress/depression for 3 months in the last year, 6) had a face disfigured by burns.

Results: The question was answered by a total of 1533 people out of the 1692 sampled. $6 \%$ of these claimed that none of the conditions stated would cause them concern. Stress/depression caused most concern to the largest percentage of people of all ages between 16 and 64 , both sexes, and all regions of the country. Only in the over $65 \mathrm{~s}$ were heart attacks and epilepsy considered as important by equivalent numbers of people. The percentages of people most concerned about mental health showed a biphasic distribution with peaks at 25-44 and 55-64. Significantly more women than men were primarily concerned about a colleague with mental health problems, but the age distribution was similar in both sexes. An overall pattern of concern emerged as: 1) mental health, 2/3) heart attack and epilepsy, 4) wheelchair use, 5) disfigurement, 6) age (very small numbers). The young and the retired age groups were relatively more concerned about physical illness and disability, and the regions showed similar patterns except that stress/depression caused significantly more concern to Londoners than to the northern English.

Conclusions: Mental health problems in work colleagues caused more concern to the majority of working age people than physical illness, disability or age. Most concern was felt by subjects who were more likely to have experienced mental health problems by reason of gender, age or region of residence.

\section{POSITIVE STEPS ON THE PATH TO EMPLOYMENT: RETURNING TO WORK AFTER MENTAL HEALTH PROBLEMS}

\section{K. Canvin, F. Ford. Department of Primary Care, Whelan Building, Univer- sity of Liverpool}

Introduction: Mental illness is the most common cause of long-term certified work incapacity, and the cause of sickness absence that causes most concern to work colleagues. Existing awareness campaigns concerning stress/depression in the workplace seem to have little impact on mental health related sickness absence. This study examines the routes to employment taken by people who have experienced mental health problems.

Method: Exploratory focus groups and in-depth interviews were conducted with people who had successfully returned to work after experiencing mental health problems. All interviews were taperecorded and transcribed verbatim. Data was analysed using grounded theory techniques. A working group comprised of people with experience of mental health problems and researchers contributed to all stages of the project, including the design of data collection and analysis.

Findings: Publicity materials were relatively unsuccessful at attracting volunteers for the study, resulting in recruitment via word of mouth and personal contacts. The resulting sample includes people who had come to terms with their experiences to an extent that they were able to disclose and discuss them. These participants successfully returned to alternative forms of work by utilising their personal coping strategies, developing skills that they used creatively in their work and viewing their adverse experiences positively. They were 'professionalising' their experiences of mental ill health, leading them work in caring, empowering, representing and campaigning roles in the field of mental health, both paid and unpaid.

External factors also influence the return to work. Family members may disable or empower the return to work. Intervention by mental health services and specialist employment services, although beneficial, seemed to lead to service dependency, enabling people to return to sheltered employment but not independent employment. Finally, workplace sickness absence procedures can influence the transition in and out of sickness absence.

Conclusions: This study uncovered a number of important and complex new areas that are fundamental to individuals' successful return to work. Positive steps and barriers on the pathway to employment are a combination of individual, structural and relationship factors. Successful interventions for the recruitment and retention of people with mental health problems must therefore be active in workplace, health care, individual and family arenas. They must also address the transferability of skills and increase the accessibility of 'conventional' work.

\section{A LONGITUDINAL ANALYSIS OF SOCIAL POSITION AND MINOR PSYCHIATRIC MORBIDITY}

R.D. Wiggins', P. Schofield, A. Sacker, J. Head, M. Bartley. Department of Epidemiology and Public Health, University College London, United Kingdom; 'Department of Sociology, City University, London, United Kingdom

Objective: To examine health inequalities in common psychiatric disorders during the period 1991-98. To assess the relative importance of social class (based on employment characteristics), social status according to the general social advantage of the household and material conditions based on annual household income.

Design: Data from 8 waves of the British Household Panel Survey, 1991-98. The relation between socioeconomic status and common mental disorders in any wave was examined using a repeated measures multilevel model.

Subjects: Men and women of working age in the UK between 1991 and 1998.

Main outcome measures: General Health Questionnaire score in any year.

Results: Among those who are economically inactive a more disadvantaged socioeconomic status increases the chances of suffering from a common mental disorder, however for those in employment this link disappears. Household income appeared to be the most closely related to psychological well being, then the general social advantage of the household (Cambridge scale) and lastly social class based on most recent employment characteristics. This social gradient is steeper when looking at those who have suffered a common mental disorder the year before.

Conclusion: There are clear relations between social class, status and income and common mental disorders however these occur among those who are economically inactive only. The results suggest 
target populations that would most benefit from interventions designed to tackle the increasingly common minor psychiatric disorders.

\section{PREDICTORS OF DEPRESSIVE SYMPTOMS IN OLDER PEOPLE: A SURVEY OF TWO GENERAL PRACTICE POPULATIONS}

T. Harris', D.G. Cook², C.R. Victor², A. Mann³ S. Shah', S. Dewilde', P. Rink', C. Beighton 1,2. 'Departments of General Practice \& Primary Care; ${ }^{2}$ Public Health Sciences, St George's Hospital Medical School, London; ${ }^{3}$ Institute of Psychiatry, Kings College, London; ${ }^{4}$ Kensington, Chelsea \& Westminster Health Authority, London

Background: Studies in older people have shown strong associations between disability, social support and depressive symptoms; but findings on the importance of socio-economic factors as independent predictors of depressive symptoms are contradictory, and little is known about the role of health locus of control.

Method: A postal survey of patients aged 65 and over registered with two London practices. The research assistant helped patients requiring assistance. Outcome measure was a score of $>5$ on the 15 item Geriatric Depression Scale (GDS15). Associations with other factors were examined using logistic regression.

Results: Response rate 75\%(1704/2287). 24.5\% (406/1655) scored $>5$ on GDS 15. Increasing age, $\geqslant 85$ vs. $65-69$ OR 3.4 (2.34.9) and female sex OR 1.7 (1.3-2.1) were related to higher depression scores. After adjusting for age, sex and practice there were strong associations between the following and higher depression scores: i) physical health and disability e.g. severe vs. no disability 26.0 (15.4-43.9); ii) social support e.g. lack of confidante OR 3.2 (2.3-4.3); iii) socio-economic e.g. manual social class OR 1.8 (1.4-2.4); iv) health locus of control e.g. internality 0.6 (0.6-0.7). After adjusting for disability, the associations between depression scores and age and sex were lost. Associations with other measures of physical health were reduced but still significant, while associations with social support, socio-economic factors, and health locus of control were unaffected.

Conclusions: Disability, physical health, social support, socioeconomic factors and health locus of control were all important depression score predictors in this group of older people. The effects of social support, socio-economic factors and health locus of control appeared independent of disability.

\section{Health behaviours}

\section{UNDERSTANDING SMOKING IN BANGLADESHI AND PAKISTANI ADULTS: IMPLICATIONS FOR SMOKING CESSATION POLICY AND PRACTICE}

J. Bush', M. White ', J. Kai ${ }^{2}$, R. Bhopal ${ }^{3}$, J. Rankin'. ' Department of Epidemiology and Public Health, University of Newcastle; ${ }^{2}$ Department of Primary Health Care and General Practice, University of Birmingham; ${ }^{3}$ Section of Public Health Sciences, University of Edinburgh

Smoking prevalence rates in some ethnic groups are high, particularly among Bangladeshi men $(47 \%$ compared to $29 \%$ average for England males). However, ethnic minority groups have not been identified as requiring special attention in national smoking cessation policies and most smoking cessation projects targeted at ethnic minority groups have taken place at a local level. Whilst several recent national and local health surveys have provided quantitative information on smoking levels in ethnic groups, there is a lack of detailed knowledge and understanding of knowledge, beliefs, attitudes and behaviours which are essential for informing the development of smoking cessation services for these groups.

Methods and results: A qualitative research project took place in Newcastle Upon Tyne between March 2000 and March 2002 which explored understandings of smoking, health and smoking behaviour in Bangladeshi and Pakistani adults. The study adopted a participatory approach and involved members of the local South Asian communities in its design, implementation and analysis. A total of 59 interviews and 36 focus groups were undertaken. The qualitative material was analysed using a grounded theory type of approach. The analysis identified four dominant, highly inter-related themes which had an important influence on smoking attitudes and behaviour: gender, age, religion and tradition. We found that smoking is a widely accepted practice in Pakistani, and particularly Bangladeshi, men and is associated with socialising and sharing. In contrast, in women smoking is traditionally associated with a sense of stigma and shame. Whilst levels of smoking in women are increasing, this activity tends to be hidden from family members. We found that young people tend to hide their smoking from elders (because of elders respected status) and peer pressure is important in influencing smoking behaviour. Interestingly, there were varied and conflicting interpretations of how acceptable smoking is within the Muslim religion. Finally, we found that the family unit plays an important role in nurturing and cultivating norms and values around smoking.

Comment: The study identified some culturally specific contexts for smoking behaviour in Bangladeshi and Pakistani adults, notably the influence of gender and religion. However, there were also strong commonalities with the UK ethnic majority, particularly in relation to age. The paper will describe how the four themes identified will help to inform national and local smoking cessation policies and practice, and in particular how such services could be targeted on the basis of gender, age, religion and tradition.

\section{THE EFFECT OF THE HOME ENVIRONMENT ON SMOKING CESSATION}

G. Norrie. MRC Social \& Public Health Sciences Unit, 4 Lilybank Gardens, Glasgow G12 8RZ

Aim: To identify factors associated with unassisted smoking cessation, in particular the role of the home environment.

Background: Recent studies have examined predictors of smoking cessation; few have focused on the home environment and its effect on an individual's smoking behaviour. This analysis includes these extra factors and identifies which are associated with smoking cessation.

Methods: The British Household Panel Study is an annual panel survey of a representative cross section of British households. The relation of socio-economic, demographic, environmental and healthrelated variables to smoking cessation was studied in 1441 self-reported smokers (657 males and 784 females) from 1125 households (14\% of subjects, $20 \%$ of households). All smokers aged 16 or above at wave 1 (1991) and present at waves 1 and 9 (1999) were included in the analysis. Logistic regression (univariate and multivariate) for the outcome of smoking cessation (non-smoker in 1999) was used to assess the influence of change in personal characteristics over the nine years (including financial status: receipt of means tested benefits, home ownership status and accumulation of savings; selfreported health status; number of dependent children). Home environmental factors were investigated with particular emphasis on the change in the spouse/cohabiting partner's smoking habits over the time period.

Results: After 9 years $28 \%$ of males and $23 \%$ of females had stopped smoking (OR 1.26, 95\% Cl 0.99-1.60). Baseline light smokers ( $\leqslant 10$ cigarettes per day) had more than twice the rate of cessation of heavy smokers (>20 cigarettes per day) with $36 \%$ stopping versus $17 \%$ (OR 2.65, 95\% Cl 1.77-3.95). Cessation occurred in $27 \%$ of subjects who lived with at least one other adult compared to only $18 \%$ of subjects who lived alone at baseline (OR 1.69, $95 \% \mathrm{Cl} 1.21-2.36$ ). After adjusting for age, gender, socio-economic status, means-tested benefits dependency and self-reported health status, the household factors single occupancy and continued smoking status of spouse continued to be associated with smoking cessation. A subject whose spouse also stopped smoking had an adjusted OR of $4.19195 \% \mathrm{Cl}$ 2.59-6.77) of quitting compared to those whose spouse never stopped smoking, while those who lived alone had an adjusted OR of 0.65 (95\% Cl 0.43-1.00).

Conclusions: Several predictors of smoking cessation were identified; in particular rates were higher when couples gave up and lower among those living alone. This should be taken into account when assessing the potential health benefits from changes in smoking behaviour and when designing interventions.

\section{PREDICTORS OF A 'PRUDENT' DIET AMONGST WOMEN AGED 20-34 LIVING IN SOUTHAMPTON}

S.R. Shore, S.M. Robinson, H.M. Inskip, S.E. Borland, D.J.P. Barker. Medical Research Council Environmental Epidemiology Unit, University of Southampton

Objectives: To identify socio-demographic predictors of diet amongst a large contemporary sample of young, non-pregnant women.

Design: The data were collected as part of the Southampton Women's Survey, a prospective study of non-pregnant women of peak child-bearing age. The interviews, conducted in the women's homes, included detailed measurements of their health and lifestyle. Diet was assessed using a food-frequency questionnaire. 
Setting: Southampton, UK

Subjects: 6129 women aged 20-34 resident in the city of Southampton and interviewed between April 1998 and June 2000. The sample was representative of the British population in terms of ethnicity, and had a similar range of Townsend index scores (a measure of deprivation).

Main outcome measure: Principal component analysis was used to summarise women's eating patterns. This statistical technique produces new variables (components), which are independent linear combinations of dietary variables with maximum variance. The first component, which discriminates most between the women, was found to characterise a 'prudent' diet; women with high scores tended to eat wholemeal bread, yoghurt, vegetables and fruit frequently, and infrequently eat white bread, full-fat milk, full-fat spread, red and processed meat, roast potatoes and chips, crisps and added sugar.

Results: Regression analysis revealed that higher educational level was the most important predictor of a high score on the prudent diet component. Each additional level of education (on a six point scale from 'none' to 'degree') increased the prudent diet score by 0.2 standard deviations. The subsequent six most important predictors of a prudent diet were, in order of importance: not smoking, watching little television, dieting, taking strenuous exercise, older age and not living with children. Having taken account of these predictors, other sociodemographic variables, including social class, made no additional important contribution. The seven characteristics together explained $32 \%$ of the variation in the prudent diet component.

Conclusions: Education was the strongest predictor of eating a prudent diet, both univariately and multivariately. This may be because women with better cognitive function are more able to control their diets, or alternatively those with higher levels of education understand and respond to health messages more readily. Whatever the reason, education appears to be particularly important in helping women to eat more appropriate diets. It is of some concern that those living with children, and presumably catering for them, are themselves eating poorer diets. That those watching more television eat a poorer diet at least indicates a suitable medium for public health dietary advice.

\section{DIET IN PRE-SCHOOL CHILDREN: WHO EATS FRUIT AND VEGETABLES?}

P. Emmett, I. Rogers, L. Glynn, S. Noble, C. Symes and The ALSPAC Study Team. Department of Child Health, University of Bristol, 24 Tyndall Avenue Bristol, BS8 ITQ, UK

Objective: To investigate factors affecting fruit and vegetable intake in young children.

Design: A prospective cohort study.

Setting: The Avon Longitudinal Study of Parents and Children (ALSPAC).

Participants: Up to 1000 children (a random sub-sample of ALSPAC) for whom 3-day dietary records were kept several times during the pre-school years.

Main outcome measures: Fruit and vegetable intakes at 18 months and $31 / 2$ years of age. Linked with information, about shopping habits and the amount of money spent on food, obtained from parents by self-completion questionnaire when their child was aged 2 years. Socio-demographic variables were obtained from questionnaires completed during pregnancy.

Results: At 18 months only $8 \%$ of children ate no vegetables during the three days of recording this had increased to $17 \%$ two years later. At both ages a similar proportion of children had not eaten fruit (around 17\%). Few children achieved the equivalent of ' 5 a day' portions of fruit and vegetables. Maternal education level was associated with the likelihood of the child consuming both fruit and fruit juice at 18 months (OR low versus high education was $0.270 p=0.001$ for fruit and $0.392 p=0.001$ for fruit juice). Younger mother were also less likely to give their child fruit or fruit juice. Although total fruit intake was related to maternal educational level vegetable intake was not. Amount of fruit and vegetables eaten by the child increased as expenditure on food increased and within each expenditure group intake rose with education level. Price appears to be a barrier to fruit intake in the lower educated groups. At $18 \mathrm{~m}$ the children of non-smokers were more likely to have eaten fruit than those of smokers (OR 1.70, $p=0.010$ adjusted for maternal education and age). They were also more likely to have eaten vegetables but this did not reach significance (adjusted OR 1.67, $\mathrm{p}=0.067$ ). Similar results were obtained at 3 years.

Conclusion: Many children consume less fruit and vegetables than recommended. The level of intake is socially patterned and may be amenable to increased education and to keeping prices down.

\section{Life course}

\section{DOES MATERNAL CHILDHOOD ENVIRONMENT AFFECT THE BIRTH WEIGHT OF HER OFFSPRING?}

E. Hyppönen', C. Power', G. Davey Smith'2. 'Centre for Paediatric Epidemiology and Biostatistics, Institute of Child Health, London; ${ }^{2}$ Department of Social Medicine, University of Bristol, Bristol

Objective: This study was undertaken to investigate the effect of mother's childhood environment (measured as growth adjusted for genetic influences) on offspring birth weight.

Design: Intergenerational population-based cohort study. Participants were followed-up regularly since birth to age 41 . Childhood height for the cohort members was measured at age 7 and 11 . Genetic growth potential for the cohort members was measured as standardised mid-parental height. Information on birth weight, gestational age and sex for children born to cohort members were obtained in 1991 and/or 1999/2000.

Setting: All births to parents born $3^{\text {rd }}$ to $9^{\text {th }}$ March 1958 in England, Scotland and Wales.

Population: Participants of the 1958 British birth cohort, their parents and their singleton first born children $(4,349$ births to female and 3,743 births male cohort members).

Results: Standardised birth weight was the strongest determinant of offspring birth weight, and robust to adjustment for mid-parental, childhood and adult height $(\beta$ for unit increase: 106 grams, $95 \% \mathrm{Cl}$ 86 to 123 ; and 81 grams, $95 \% \mathrm{Cl} 58$ to 104 , for females and males, respectively). Mother's (i.e. female cohort member's) standardised height at 7 years ( $\beta$ for unit increase: 36 grams, $95 \% \mathrm{Cl} 9$ to 62 ), but not in adulthood ( $\beta$ for unit increase 18 grams, $95 \% \mathrm{Cl}-11$ to 47 ), was associated with offspring birth weight even after adjustment for other growth measures. Father's childhood height and adult height were associated with offspring birth weight when adjusted for sex and gestational age of the child, but not when controlled for cohort member's previous or subsequent growth measures. Observed associations were largely robust to adjustment for cohort member's social class at birth and in adulthood.

Conclusions: These results suggest a key role for mother's early environment as a determinant of her subsequent reproductive function. Our observation stresses the importance of optimising the modifiable childhood conditions, not only for the benefit of a mother's own health, but also for ensuring a good starting-point for the next generation.

\section{BIRTH WEIGHT IN OFFSPRING AND INSULIN RESISTANCE IN LATE ADULTHOOD: BRITISH WOMEN'S HEART AND HEALTH STUDY}

D.A. Lawlor', G. Davey Smith', S. Ebrahim'. 'Department of Social Medicine, University of Bristol

Background: Low birth weight individuals not only have increased risk of cardiovascular disease themselves, but their parents are also at increased risk. While the former association may be explained by a programming effect of the intrauterine environment, the latter must have a different explanation, since it is inconceivable that an infant's low birth weight can determine its own parents' risk of cardiovascular disease through intrauterine programming. A plausible explanation for these trans-generational associations is found in the foetal insulin hypothesis. This proposes that polymorphisms exist that determine both insulin resistance in adult life and, impaired insulin-mediated growth in the foetus, resulting in low birth weight. If the foetal insulin hypothesis is correct then offspring birth weight should be associated with parental insulin resistance.

Design: Cross sectional survey.

Participants: 4286 women aged 60-79 years from 23 towns across England, Scotland and Wales.

Main outcome measures: Maternal insulin resistance.

Results: Offspring birth weight was inversely associated with maternal insulin resistance in older age. There was a U-shaped association between offspring birth weight and maternal diabetes in older age, with women in the lowest and highest quartiles of offspring birth weight having the highest prevalence of diabetes. For each $1 \mathrm{~kg}$ higher offspring birth weight the odds of the mother being in the highest compared to all other quartiles of HOMA score (insulin resistance) was $15 \%$ lower lodds ratio $0.85 \%, 95 \%$ confidence interval $0.71-1.00$ ). This strengthened to $27 \%$ (odds ratio $0.73 ; 0.60-0.90$ ) on adjustment for potential confounding factors lage, body mass 
index, systolic and diastolic blood pressure, high density lipoprotein cholesterol, low density lipoprotein triglycerides, smoking, adult and childhood social class).

Conclusions: Offspring birth weight is inversely associated with maternal insulin resistance in late adulthood. This is despite impaired glucose tolerance during pregnancy being associated with higher birth weight offspring. This finding supports the hypothesis that common genetic factors associated with both insulin resistance and insulin mediated foetal growth contribute to the association between birth weight and adult cardiovascular disease and diabetes risk.

\section{EARLY EMERGENCE OF RISK OF TYPE 2 DIABETES AND CARDIOVASCULAR DISEASE AMONG SOUTH ASIANS: NEW EVIDENCE FROM A STUDY OF ADOLESCENTS}

P.H. Whincup', J.A. Gilg', K. Odoki', P.M. Ueland'2, H. Refsum², K.G.M.M. Alberti ${ }^{3}$, D.G. Cook'. 'Department of Public Health Sciences, St George's Hospital Medical School, London; ${ }^{2}$ Locus for Homocysteine and Related Vitamins, University of Bergen, Norway; ${ }^{3}$ Department of Diabetes, University of Newcastle-on-Tyne

Objective: To examine whether glucose metabolism, insulin resistance, adiposity and other cardiovascular risk factors differ between British adolescents of South Asian and European origin.

Design: School-based cross-sectional study (1998-2000)

Setting: Secondary schools in ten towns in England and Wales.

Participants: 168 South Asian and 2422 European pupils aged $12-16$ years $(67 \%$ response), with fasting blood samples collected from 1507 of the older pupils (69\% response).

Outcome measures: Body mass index, waist-hip ratio, skinfold measurements, fasting glucose and insulin, blood pressure, blood lipids.

Results: Compared with Europeans, South Asian children had a lower mean body mass index (percentage difference $4.0,95 \% \mathrm{Cl} 1.1$ to $7.1 \%$ ), a similar mean waist-hip ratio but a higher subscapular:triceps ratio (mean difference $0.098,95 \% \mathrm{Cl} 0.060,0.136$ ). Mean fasting plasma glucose level was higher among South Asians than Europeans (mean value 5.20 vs $5.06 \mathrm{mmol} / \mathrm{L}$; mean difference 0.15 $\mathrm{mmol} / \mathrm{L}, 95 \% \mathrm{Cl} 0.04$ to 0.25 ); the prevalence of impaired fasting glucose or fasting hyperglycaemia $(\geqslant 6.1 \mathrm{mmol} / \mathrm{L})$ was higher among South Asians (5.6\% vs 1.5\%, $p=0.02$ ). Fasting insulin levels were higher among South Asian children than Europeans (percentage difference $12.0 \%, 95 \% \mathrm{Cl} 1.4$ to $21.5 \%$ ); the difference (seen in both sexes) was increased after adjustment for height, body mass index and pubertal status, though attenuated slightly after adjustment for plasma glucose. Mean systolic pressure was lower in South Asians (mean difference $3.6 \mathrm{mmHg}, 95 \% \mathrm{Cl} 1.2$ to $5.9 \mathrm{mmHg}$ ) despite a higher mean pulse rate (mean difference $3.9 / \mathrm{min}, 95 \% \mathrm{Cl} 1.6$ to $6.1 / \mathrm{min})$. Total, LDL, HDL cholesterol and triglyceride levels were similar in both groups. South Asians had lower vitamin C levels (mean difference $10.6 \mu \mathrm{mol} / \mathrm{L}, 95 \% \mathrm{Cl} 5.4$ to $15.8 \mu \mathrm{mol} / \mathrm{L}$ ) and lower folate levels (percentage difference $20 \%, 95 \% \mathrm{Cl} 5.3$ to $37.0 \%$ ) though homocysteine levels were similar in both groups.

Conclusions: The predisposition to type 2 diabetes and insulin resistance observed in South Asian adults is also apparent among adolescents. Establishing the contributions of the childhood and fetal environments and of genetic factors to the development of these differences is an important priority. Effective strategies for prevention of type 2 diabetes and cardiovascular disease in British South Asians may need to begin in childhood or earlier.

\section{SOCIAL INEQUALITIES IN HEIGHT: LIFECOURSE AND INTER-GENERATIONAL TRENDS}

L. Li', O. Manor', C. Power'. 'Centre for Paediatric Epidemiology and Biostatistics, Institute of Child Health, London; ${ }^{2}$ School of Public Health and Community Medicine, Hebrew University, Israel

Objectives: To investigate social inequalities in height within and across generations, in order to establish whether inequalities vary by life stage and whether they have changed between two generations.

Design: 1958 British birth cohort and one-third of their offspring.

Setting: England, Scotland and Wales.

Participants: All children born 3rd-9th March, 1958, followed to 1991 , and their offspring.

Main outcome measures: Height at ages 7, 11, 16, and 33y for cohort members. One height measure at age 4-18y for the offspring.

Methods: Multilevel linear models were applied to examine (a) the association between social class of origin (at age 7) and the child-toadult growth trajectory for cohort members and (b) associations of social class with height of offspring and their height gain relative to cohort members.
Results: Social inequalities in height were observed among cohort members, with differences $>2.0 \mathrm{~cm}$ at all ages between classes I\&II and IV\&V. The effect of social class reduced, but remained significant at all ages after adjusting for parental height. Social class was also associated with growth tempo: boys from classes IV\&V were shorter in childhood, but grew faster throughout adolescence and continued to grow after age 16y; girls from classes IV\&V had a faster growth rate until age $11 y$, but then grew at a slower rate, maturing later than girls from classes I\&II. By adulthood, the class difference in mean height had reduced slightly because girls from classes IV\&V either grew more rapidly or for a longer period.

A secular increase in height was seen among the offspring relative to cohort members, which was mainly due to a greater height gain in classes IV\&V. Male offspring had a similar height on average to their fathers in classes I\&II, while offspring in classes IV\&V gained $2.1 \mathrm{~cm}$ $(p<0.001)$. Height gains of female offspring relative to their mothers were evident in all classes, though a greater gain in classes IV\&V was non-significant. Thus social class effect on height was weaker in the offspring generation, with a difference between classes I\&II and IV\&V of $0.7 \mathrm{~cm}$.

Conclusions: An effect of social class on height observed among a cohort born in 1958 had weakened substantially in the offspring generation. The significant narrowing of class inequalities was due to a greater height gain among offspring from manual social backgrounds. The causes of this reduced inequality require further clarification.

\section{Policy and population health}

\section{FLUORIDATION AND TOOTH EROSION IN 14-YEAR OLD SCHOOLCHILDREN}

S. Taylor', A. Milosevic', P. Bardsley'. 'Division of Statistics and O.R., Department of Mathematical Sciences, University of Liverpool; ${ }^{2}$ School of Clinical Dental Sciences, University of Liverpool

Objectives: Although there is a body of evidence for the protective role of water fluoridation against dental caries, its association with tooth erosion has not been researched. The aim of this study was to assess the aetiological role of water fluoridation in the acid erosion of teeth. Other factors assessed were social status, dental care and dietary habits. The study has a clustered sampling structure: subjects within schools within districts. Random effects modelling techniques were used to investigate aetiology, as these can accommodate the effects of clustering within a sample and produce more statistically efficient results.

Data: The data were collected by the Department of Clinical Dental Sciences at the University of Liverpool. A sample of 2385 children were randomly selected from 56 schools and 12 districts in North-West England. Tooth erosion was assessed on three surfaces of the six upper and lower anterior (front) teeth. Individuals were simply dichotomised as those with or without tooth erosion. A 34-item questionnaire assessed aspects of general and dental health, diet and dental knowledge.

Results: The sampled population had $9.8 \%$ subjects identified as cases of tooth erosion. Of these, $7.7 \%$ lived in non-fluoridated residences and $2.1 \%$ in fluoridated (univariate chi-square $=4.33, p=$ 0.038 ). There was significant variation in erosion between districts and between schools. None of the 20 acidic food and drink items showed a significant association with erosion. Food and drink items were not included in subsequent regression analysis.

Multilevel logistic regression models showed no effect of fluoridation when controlling for other factors. More frequent tooth brushing was associated with an increased risk of erosion, but had a significant interaction with water fluoridation that reversed this association.

Conclusions: The study shows there is considerable variation in dental erosion between subjects, districts and schools. The aetiological factors investigated could not explain much of this, but there is indication that the combined effect of fluoridation and regular tooth brushing may be protective. The results of this study suggest that other factors may need to be explored to explain variation in acid erosion of teeth among schoolchildren. 


\section{EFFECT OF ERADICATION OF HELICOBACTER PYLORI INFECTION IN THE COMMUNITY ON THE INCIDENCE OF DYSPEPSIA}

J.A. Lane', L.J. Murray', I.M. Harvey', J.L. Donovan', M. Egger', R.F. Harvey'. 'Department of Social Medicine, University of Bristol; ; ${ }^{2}$ Queens University of Belfast, Belfast; ${ }^{3}$ Universtiy of East Anglia, Norwich; ${ }^{4}$ Frenchay Hospital, Bristol

Introduction: Eradication of $H$ pylori infection may reduce dyspepsia, peptic ulcer complications and health resource utilization. We have investigated the impact of an $H$ pylori eradication programme on the incidence of dyspepsia in the community, in a large double blind randomised controlled trial.

Design: Double blind randomised placebo controlled trial.

Subjects and setting: Unselected community population aged 20-59 years in south west UK.

Intervention: Participants infected with $H$ pylori randomised to ranitidine bismuth citrate $400 \mathrm{mg}$ and clarithromycin $500 \mathrm{mg}$ twice daily for 2 weeks or placebo.

Main outcome measures: Consultation rates in primary care for dyspepsia, symptoms and health care utilization for dyspepsia.

Methods: All patients aged 20-59 years registered with seven primary care centres $(N=26,203)$ were invited to take part in the project. Participants completed validated dyspepsia and quality of life questionnaires and $\mathrm{H}$ pylori infection was assessed by the ${ }^{13} \mathrm{C}$ urea breath test (UBT). Infected participants were randomised to receive either ranitidine bismuth citrate $400 \mathrm{mg}$ and clarithromycin $500 \mathrm{mg}$ twice daily for 2 weeks or placebo. A blinded second UBT was carried out at 6 months and further questionnaires were completed after 2 years.

Results: 10,537 participants were recruited and 1634 (15.5\%) were positive for $\mathrm{H}$ pylori. 1558 participants $(95 \%)$ were randomised and two year follow-up was obtained on 1438 participants (92\%). H pylori infection was successfully eradicated in $90.7 \%$ of those given active therapy $(727 / 787)$. By intention to treat analysis, 159/787 $(20.2 \%)$ of those who received active therapy had dyspepsia (epigastric pain) regularly at two years, compared with $202 / 771(26.2 \%)$ of those given placebo, odds ratio $=0.68(95 \% \mathrm{Cl} 0.53,0.87)$. Primary care consultations for dyspepsia were $30 \%$ less in the eradication arm (55 participants over 2 years compared with 78 participants in the placebo arm).

Conclusion: $H$ pylori eradication leads to a $6 \%$ reduction in dyspepsia symptoms in a community screening and eradication trial, potentially resulting in health improvements and large cumulative savings in health expenditure.

\section{IMPACT OF AN INTERVENTION TO REDUCE OUTDOOR AIR POLLUTION ON HOSPITAL ADMISSIONS FOR ASTHMA IN CHILDREN}

B. Olowokure', P.J. Saunders' ${ }^{2}$ R.C. Wilson ${ }^{2}$, R.L. Smith ${ }^{3}$. 'Department of Public Health, Sandwell Health Authority, UK; ${ }^{2}$ Department of Public Health and Epidemiology, University of Birmingham, UK; ${ }^{3}$ West Midlands Cancer Intelligence Unit, University of Birmingham, UK

Background: Over a number of years the Sandwell Department of Public Health UK has received complaints from parents and teachers regarding emissions from a nearby foundry. They were particularly concerned about the possible adverse effects of the emissions on children's respiratory health. In 1997 foundry management introduced an intervention designed to reduce foundry emissions. This study assessed whether there was an excess of respiratory ill health in children in the area around the foundry and whether changes occurred in respiratory health following introduction of the intervention.

Methods: An ecological study employing a concentric circle approach was used to define the study area. A geographical information system was used to construct a series of nine concentric $250 \mathrm{~m}$ bands centred on the foundry. A line was drawn through the centre of the foundry, from north to south, dividing the study area into two areas of probable exposure to air pollution based on the prevailing wind direction. These were designated as high-risk area A (downwind) and low-risk area B (upwind). Impact of the intervention was assessed by examining emergency hospital admission rates for childhood asthma. The chi-square test was used to test the association between hospital admission rates in the 2-years before (1995/96$1996 / 97$ ) the intervention was introduced, and 2-years after (1998/ 99-1999/00) the intervention had been operating for one year in children aged 0-14 years.

Results: In the 2-years before the intervention was introduced overall hospital admissions for children in area $A$ were 1.8 times the rate in area $\mathrm{B}(95 \% \mathrm{Cl} 1.3-2.4 ; \mathrm{p}=0.00025)$. Following the intervention overall hospital admission rates for area $A$ were 1.3 times that seen in area $B(0.8-2.0 ; p=0.41)$. More detailed examination by 5 -year age groups revealed that in area $A$ significant reductions in admission rates were seen for children aged $0-4$ years $\left(19.6 / 10^{3}\right.$ to $5.3 / 10^{3}$. $p<0.00001)$ and those aged $5-9$ years $\left(9.0 / 10^{3}\right.$ to $4.5 / 10^{3}$; $\mathrm{p}<0.038$ ) after the intervention. In area $\mathrm{B}$ the only significant reduction was seen in the $0-4$ year olds $\left(9.8 / 10^{3}\right.$ to $4.0 / 10^{3}$; $\mathrm{p}<0.0065)$. Overall, significant trends were not identified in relation to distance from the foundry.

Conclusions: Our results suggest that a significant reduction has occurred in admission rates for asthma following the intervention at the foundry. The greatest benefit appears to have been in children aged $0-4$ years and in areas downwind from the foundry. However, the limitations of this type of study need to be acknowledged especially small numbers and possible individual-level confounders.

\section{THE HEALTH IMPACTS OF NEW ROADS: A SYSTEMATIC REVIEW}

M. Egan, V. Hamilton, M. Petticrew, D. Ogilvie. MRC Social and Public Health Sciences Unit, University of Glasgow

Objective: To review the available evidence on the positive and negative impacts of new road building on human health and wellbeing.

Design: Systematic review of primary intervention studies measuring health outcomes related to new roads.

Source: Extensive literature survey to locate studies in any language or format, identified from social science, transport, medical, and grey literature databases, personal collections, expert consultation, and reference lists.

Main outcome measures: Accident injuries, community severance, noise, dirt, and ambient pollution.

Results: The best available evidence suggests that the introduction of new bypasses significantly decreases accident injuries per vehicle $\mathrm{km}$ travelled. New arterial roads in urban areas do not appear to have a significant impact on accident injury rates. However, widening existing arterial roads can significantly reduce accidents if certain safety measures are included in the improvements.

Bypasses decrease perceptions of community severance within the area being bypassed. Arterial roads increase perceptions of severance. In the short term, changing perceptions of severance do not appear to lead to changes in social behaviour likely to encourage or hinder social network formation. The long-term effects of new roads on perceived severance, social networks, and health require further research.

Annoyance related to noise, dirt, and ambient pollution is closely related to traffic density: new urban arterial roads increase annoyance, whilst bypasses decrease annoyance. There is a lack of evidence regarding specific health outcomes related to pollution from new roads, although there is some evidence that new roads do not affect the incidence of asthma.

Conclusion: Whilst there have been many studies investigating the environmental impact of new roads, few have attempted to measure human health outcomes. Bypasses have a more positive impact on health than arterial roads, particularly in decreasing accident rates. However more research is needed to better understand the impacts of pollution and community severance associated with new roads. The results of this review will be discussed in the context of health impact assessment (HIA), and of healthy public transport policy.

\section{Inequality II}

\section{DOES GETTING A DEGREE ELIMINATE THE INFLUENCE OF CHILDHOOD SOCIO-ECONOMIC STATUS ON ADULTHOOD SELF-RATED HEALTH PROBLEMS AND DISABILITY?}

W.A. Markham', P. Aveyard', A. Bullock', H. Thomas'. 'School of Education, University of Birmingham, Birmingham; ${ }^{2}$ Department of Public Health \& Epidemiology, University of Birmingham, Birmingham

Objectives: To examine whether childhood socio-economic status constituted a risk factor for adulthood self-rated health problems and disability even among those graduating from university.

Design: Retrospective cohort study comparing graduates from different childhood social classes. The influence of childhood social class 
was examined as a linear trend in a logistic regression equation predicting disability in 1996 .

Participants: 3630 people graduating in 1985 and 5624 people graduating in 1990 from 20 UK degree-awarding higher education institutions.

Main outcome measures: Self-rated health problems and disability.

Results: In the youngest age group (25 years), there was no gradient in disability by childhood social class, but the gradient became increasingly steep in older graduates. By age 38 (the oldest) the prevalence of disability was $3.4 \%$ in graduates from social class in childhood, and $10.0 \%$ in graduates from social class $V$ in childhood. Adjustment for markers of persistent childhood disability and adulthood socio-economic status 1, 6, and 11 years after graduation did not greatly influence the risk.

Conclusions: If anyone can escape the influence of poverty in childhood it should be graduates, who are highly educated and usually attain high social status and relative wealth. This study indicates that whatever the nature of the biological and/or psychological effects of low childhood socio-economic status on adult health, they are not offset by education or income.

\section{CHILDHOOD IQ, SOCIAL CLASS, DEPRIVATION AND THEIR RELATIONSHIPS WITH MORTALITY AND MORBIDITY RISK IN LATER LIFE: LINKING THE SCOTTISH MENTAL SURVEY 1932 AND THE MIDSPAN STUDIES}

C.L. Hart', I. J. Deary², D.J. Hole', G. Davey Smith33, M.D. Taylor², L.J. Whalley', J.M. Starr' ${ }^{5}$, V. Wilson'. 'Department of Public Health, University of Glasgow; ${ }^{2}$ Department of Psychology, University of Edinburgh; ${ }^{3}$ Department of Social Medicine, University of Bristol; ${ }^{4}$ Department of Mental Health, University of Aberdeen; ${ }^{5}$ Department of Geriatric Medicine, University of Edinburgh; ' ${ }^{\circ}$ Scottish Council for Research in Education

Objectives: The purpose of the study was to investigate mortality and morbidity risk in relation to childhood $I Q$, social class and deprivation in people born in 1921

Design: Prospective cohort studies conducted on adults in the 1970s, with retrospective data from childhood.

Setting and subjects: Participants were from two of the MIDSPAN studies - the Collaborative study, located in 27 workplaces in the central belt of Scotland and the Renfrew/Paisley general population study of residents from those towns. 938 Midspan participants were successfully matched with the Scottish Mental Survey 1932 in which almost all 1921-born people attending Scottish schools on 1 June $1932(N=87,498)$ took a cognitive ability test.

Main outcome measures: Hospital admissions and cancer registrations (obtained by computerised linkage with the Scottish Morbidity Records system) and mortality, in a 25 year follow up period.

Results: Childhood IQ was related to social class and deprivation category of residence in adulthood, with a decrease in $I Q$ seen with worsening social class and deprivation. In 25 years, 432 participants died. The risk of dying was $17 \%$ higher for each standard deviation (15) disadvantage in childhood IQ. Adjustment for social class and deprivation category accounted for some, but not all of this higher risk, reducing the risk to $12 \%$. Analysis by $I Q$ quartile showed increased risk of death for the lowest quarter only. There was an $11 \%$ risk of dying for each lower social class. Adjustment for IQ reduced this to $6 \%$ and additional adjustment for deprivation reduced it to $4 \%$. There was an $11 \%$ risk of death per deprivation category, which was reduced to $8 \%$ by adjustment for IQ and for both IQ and social class. Cause specific mortality or hospital admission showed higher risks for all cardiovascular disease, coronary heart disease, respiratory disease and stroke with lower childhood IQ. For cancers, cause specific mortality or cancer registration risk was higher with decreasing childhood IQ for all cancer, lung cancer and stomach cancer, and lower for colorectal and female breast cancer. Adjustment for social class and deprivation had a small effect on the cause specific risks.

Conclusions: Lower childhood IQ was related to higher mortality risk and certain causes of death or morbidity. This unique data set has shown that childhood IQ can be considered as a marker for death or illness in later life in similar and complementary ways to social class or deprivation category.

\section{MODELLING SOCIAL INEQUALITIES IN HEALTH OVER TIME: A LATENT CLASS AND LATENT GROWTH CURVE APPLICATION}

R.D. Wiggins', A. Sacker', M. Bartley2. 'Department of Sociology, City University London, Northampton Square, London, ECIV OHB, UK ${ }^{2}$ Department of Epidemiology and Public Health, University College London, 1-19 Torrington Place, London WCIE 6BT, UK

Objectives: This paper employs a combination of latent class (LCA) and latent variable methods to model latent health status over time for a set of observed binary variables. It is motivated by a desire to move forward from the analysis of health inequalities based on repeated cross-sectional data to an examination of the development of inequalities in health over time.

Design: In the British Household Panel Survey (BHPS), there is a checklist of 13 health problems asked annually since 1991. Using repeat measures for 8 waves of data, we first present findings for a set of health indicators using latent class analysis (LCA) under the WinLTA program. Health scores are calculated at the individual level from the LCA estimated probabilities of class membership and class frequencies. The health scores are next entered into a structural equation model of the change in health status over the time period 1991. 1998. This growth model is then further extended to incorporate the relationship between socio-economic position and health using a multigroup analysis. Altogether seven social groups were compared, based on the Office for National Statistics new socio-economic classification (ONS-SEC). All parameters were estimated using FIML (implemented in AMOS) to compensate for missingness.

Subjects: Adult participants in the British Household Panel Survey aged 35 to 60 years in 1991

Outcome measure: Latent health scores.

Results: A quadratic growth model fitted the data well. The mean values of the intercept in the multigroup ONS-SEC model were consistent with previous cross-sectional reports of health inequalities: mean health scores in 1991 were poorer for those in more disadvantaged social positions than their more advantaged peers. Overall, health deteriorates faster for those with better initial health status. However, on average, deterioration was still relatively faster among those in the most disadvantaged social positions over the 8-year period irrespective of their poorer initial health scores.

Conclusion: Latent variable growth models are a valuable tool for understanding the dynamics of social inequalities in health.

\section{TREND EFFECTS OF SOCIO-ECONOMIC POSITION AND MOBILITY ON HEALTH-RELATED OUTCOMES IN THE MIDSPAN FAMILY STUDY}

A. McConnachie', K. Hunt², M.N. Upton ${ }^{3}$, C.L. Hart ${ }^{4}$, G. Davey Smith ${ }^{3}$, G.C.M. Watt'. 'Department of General Practice, Glasgow University; ${ }^{2}$ MRC Social and Public Health Sciences Unit, Glasgow; ${ }^{3}$ Department of Social Medicine, University of Bristol; ${ }^{4}$ Department of Public Health, Glasgow University

Objective: To investigate health related correlates of relative socio-economic mobility.

Design: Two cross sectional studies: the MIDSPAN Study (1972-6) and the MIDSPAN Family Study (1996).

Setting: Renfrew and Paisley, West of Scotland

Participants: 2,338 adult (aged 30-59) offspring (screened in 1996) from 1,477 married couples (screened in 1972-6).

Main outcome measures in offspring: Height, BMI, waist/hip ratio, SBP, total/HDL cholesterol ratio, \%-predicted $\mathrm{FEV}, \%$ food energy intake from fat, current smoking, current alcohol intake, regular physical activity, prevalent limiting long-standing illness, prevalent cardiovascular disease, prevalent asthma and/or wheeze.

Results: Most outcomes were associated with socio-economic position (defined using a composite measure of occupational social class, area-based deprivation, housing tenure and vehicular access), either in terms of original (childhood) or achieved (adult) position, with those in or from higher positions tending to be healthier. Outcomes showing little or no social gradient were SBP (men and women), BMI (men), total/HDL cholesterol (men) and high alcohol intake (women). Adjusting for original socio-economic position, most outcomes were related to the degree of relative social mobility (defined in terms of the relative rank of individuals' and their fathers' socio-economic positions within each population), with those who had been most upwardly mobile tending to be healthier. However, adjusting for current socio-economic position, mobility was generally unrelated to outcomes, with the exceptions of \%-predicted FEV and regular physical activity. Lower childhood socio-economic position was associated with impaired lung function and being less likely to take regular exercise in adulthood. Whilst men and women showed differing socio-economic gradients in health-related behaviours, they showed similar associations with relative social mobility.

Conclusions: Direct associations between health-related outcomes and childhood socio-economic position are uncommon in this cohort; in general, social patterns in health are due to associations with current position. This could be a result of one or both of: (a) health selection, whereby the healthiest individuals achieve higher socioeconomic positions; or (b) social causation, whereby adverse socio-economic conditions cause ill health. The observation that 
\%-predicted FEV, showed an association with socio-economic mobility after adjusting for current position supports the hypothesis of early life influences on lung development. Habitual physical activity may also be influenced by early socio-economic environment. Differing socio-economic gradients between men and women in health-related behaviours, despite similar associations with mobility may be an artefact of classifying women's childhood circumstances by paternal socio-economic position, but may also indicate different degrees of social causation between the sexes.

\section{MORTALITY OF MIGRANTS FROM THE INDIAN SUB-CONTINENT AND DURATION OF RESIDENCE IN ENGLAND AND WALES}

S. Harding. MRC, Social and Public Health Sciences Unit, University of Glasgow, Glasgow, Scotland

Aim: To examine mortality of South Asian migrants by duration of residence in England and Wales.

Design: Longitudinal study, 1971-99, of south Asian migrants in the 1971 Census aged 25 to 54 years. Duration of residence was measured using year of arrival and related to mortality using Cox regression. Socio-economic position was measured using occupational social class and access to cars.

Main results: All-cause mortality of south Asians who arrived earlier was higher than that of those who arrived later. At ages 25-34 in 1971,5 yearly increases in duration of residence was associated with a hazard ratio of 1.46 (95\% confidence interval 1.14-1.87, deaths $113)$, and at ages 35-44 with $1.21(1.03-1.42,200)$. Cardiovascular disease mortality was the main attributable cause to this trend. At ages 25-34 in 1971, 5 yearly increases in duration of residence was associated with a hazard ratio of $1.55(1.13-2.13,65)$, and at ages $35-44$ with $1.26(1.01-1.57,103)$. For cancers, a significant relationship with duration of residence was seen at ages 35-44 (hazard ratio $1.54,1.05-2.26,31)$ and at ages $45-54(1.30,1.07-1.57,24)$. This trend remained after adjusting for differences in socio-economic position by duration of residence.

When mortality of south Asians was compared with all other study members, excess all cardiovascular mortality adjusted for socioeconomic differences at ages 25-34 and 35-44 was observed for only the long established residents.

Conclusion: This study shows evidence of a positive relationship between mortality from cardiovascular disease cancers, and duration of residence in England and Wales among South Asians.

\section{CVD treatment}

\section{IS CORONARY REVASCULARISATION UNDERUSED AMONG OLDER PEOPLE? PROSPECTIVE FINDINGS FROM THE ACRE STUDY}

S. Gnani', N.K. Fitzpatrick', A.M Crook', A. Timmis², P.G. Magee², H. Hemingway', . 'Department of Research and Development, Westminster Primary Care Trust, 50 Eastbourne Terrace, London W2 6LX; ${ }^{2}$ Cardiac Directorate, Barts and the London NHS Trust, Bonner Road, London E2 9JX: ${ }^{3}$ Department of Epidemiology and Public Health, University College London Medical School, 1-19 Torrington Place, London WC1E 6BT

Objective: To determine whether coronary revascularisation is underused in older patients, using appropriateness ratings as a marker of clinical need.

Design: Prospective cohort study with 2.5 years follow up. A nine member expert panel rated the appropriateness of revascularisation independently of patient age.

Setting: Tertiary cardiac centre in the City and east London.

Patients: 4020 consecutive patients undergoing coronary angiography in the Appropriateness of Coronary Revascularisation (ACRE) study. There were no exclusion criteria. There were 2601 patients aged $<65$ years, 1162 aged $65-74$ years and 250 aged $75-84$ years; median age 62 years.

Main outcome measures: Coronary revascularisation, all cause mortality

Results: Among the 1353 patients in whom CABG was deemed appropriate, older compared to younger patients were equally likely to undergo CABG: patients aged $75-84$ years vs $<65$ years hazard ratio 1.29 , $(95 \%$ confidence interval(Cl) $0.97-1.71)$. All cause mortality was higher among patients for whom $C A B G$ was deemed appropriate but who underwent medial management compared to those who received CABG: the hazard ratios $(95 \% \mathrm{Cl} 1.60-5.01)$ in patients aged $<65$ years, $4.62(95 \% \mathrm{Cl} 2.54-8.40)$ in patients aged $65-74$ years and $7.29(95 \% \mathrm{Cl} 2.19-24.23)$ in patients aged $75-84$ years after adjustment for number of diseased vessels, left ventricular function and Parsonnet operative risk score. The absolute risk differences in these three age groups were $8.3 \%, 18.3 \%$ and $35.3 \%$ respectively. Coronary angioplasty among patients in whom it was deemed appropriate, was performed at a lower rate in patients aged $65-74$ years compared to patients aged $<65$ years, hazard ratio 0.69 (95\% Cl 0.54-0.90).

Conclusions: The mortality impact of underuse of CABG may be greater among older patients in relative and absolute terms. With the lack of randomised trials of revascularisation among older age groups, appropriateness ratings offer a useful method of investigating age inequalities.

\section{WHAT ARE THE BENEFITS ASSOCIATED WITH MEETING THE NATIONAL SERVICE FRAMEWORK TARGETS FOR ACUTE MYOCARDIAL INFARCTION?}

D. Chase', P. Roderick', K. Cooper', R. Davies ${ }^{2}$, J. Raftery ${ }^{3}$. 'Health Care Research Unit, Southampton General Hospital; ${ }^{2}$ School of Management, University of Southampton; ${ }^{3}$ Health Services Management Centre, Birmingham

Background: Standards of care for ambulance response and thrombolysis timing were set out in the National Service Framework for CHD in 2000. Namely, that by April 2001, call-to-needle time for thrombolysis for acute myocardial infarctions would be reduced by (1) improving ambulance response times so that $75 \%$ of category A calls receive a response within 8 minutes and (2) enabling $75 \%$ of eligible patients to receive thrombolysis within 30 minutes of hospital arrival by April 2002 and within 20 minutes by April 2003. There have been no published quantitative assessments of the potential health gains, and likely cost, of meeting these targets. The aim of this study was to quantify the health gains and costs associated with moving from current practice to the NSF targets shown above using a computer simulation model.

Methods: A computer simulation model of the epidemology, prevention and treatment of coronary heart disease (CHD) in an English population has been developed. The model outputs are numbers of people, disease events and interventions for each risk and disease category for each year. Simulated health care activity is linked to data on unit costs so that different intervention scenarios can be costed and costs per life year saved derived. Costs are discounted at $6 \%$ and benefits at $1.5 \%$. A simulation was run through the treatment module of the CHD model for a population of 25 million for 20 years.

Results: Attaining the ambulance response NSF target resulted in 9 deaths prevented which translates into 85 life years saved for a population of one million for an average year, with a cost per life year saved of between $£ 3,400$ and $£ 24,300$. The benefit of meeting the thrombolysis NSF target will be presented. Other scenarios will also be presented e.g. Maximising ambulance response and increasing the number of patients administered thrombolysis.

Conclusions: Meeting the NSF target for ambulance response appears to be cost-effective especially as other benefits of faster ambulance response have not been considered. However, the number of lives saved is less than previous estimates. It has been suggested that a radical service change would be required to meet the thrombolysis NSF target. Further substantial increases in lives saved would require a reduction in the time between onset of symptoms and call to emergency services.

\section{USE OF STATINS IN THE SECONDARY PREVENTION OF CHD: IS TREATMENT EQUITABLE?}

F.D.A. Reid, D.G. Cook, P.H. Whincup. Department of Public Health Sciences, St George's Hospital Medical School, Cranmer Terrace, London SWI7 ORE

Objective: To investigate possible inequities in the use of statins for people with coronary heart disease, according to a wide range of social and clinical factors.

Design and setting: Cross-sectional analysis of data from the Health Survey for England 1998, a population based survey.

Subjects: 760 adults with coronary heart disease (CHD).

Results: Only $19.9 \%$ of subjects with CHD were receiving lipid lowering medication (151/760; $95 \% \mathrm{Cl} 17.0 \%$ to $22.7 \%)$. The likelihood of receiving statins was greatly reduced for older age groups: compared to those aged $<65$ years, the odds of receiving statin treatment was 0.53 for subjects aged $65-74$ years $(95 \% \mathrm{Cl} 0.35$ to 0.80$)$, 
and 0.11 for subjects aged 75 years and over $(95 \% \mathrm{Cl} 0.06$ to 0.21$)$. Statin treatment was lower for current cigarette smokers compared to non-smokers (odds ratio $0.55 ; 95 \% \mathrm{Cl} 0.32$ to 0.96 ), and for subjects with angina compared to those with a previous myocardial infarction (odds ratio $0.63 ; 95 \% \mathrm{Cl} 0.43$ to 0.93 ). Lower levels of statin use were also seen with increasing time since diagnosis $(p=0.12)$. No clear associations were observed with social measures.

Conclusions: Important inequalities were found in the use of statins among people with CHD, which could not be justified by evidence from the large statin trials. Proactive policies are required to ensure that the vast majority of (if not all) patients with CHD are receiving statins, regardless of age, sex, social class, smoking status, type of $\mathrm{CHD}$, or time since diagnosis.

\section{IDENTIFYING AND EXPLAINING VARIATION IN LIPID LOWERING DRUG USE USING THE DOCTORS' INDEPENDENT NETWORK DATABASE}

S. DeWilde',2, I.M. Carey ${ }^{1,2}$, S.A. Bremner ${ }^{1,2}$, N. Richards', S.R. Hilton', D.G. Cook ${ }^{2}$ ' Department of General Practice \& Primary Care; ${ }^{2}$ Department of Public Health Sciences, St George's Hospital Medical School, London SW17 ORE; ${ }^{3}$ CompuFile Ltd., Surrey GU23 7EF

Background: Recent research has indicated that there is a low use of lipid lowering drugs (LLD) in people with Ischaemic Heart Disease $(\mathrm{HDD})$, and that women are less likely to receive such drugs than men. We examined trends in the prescription of LLD in subjects with IHD between 1994 and 2001 and sought explanations for variations in prescribing in 1998.

Setting: The Doctors' Independent Network (DIN) database consists of routinely entered primary care data from practices in England and Wales using the Meditel (Torex) System 5. The present study is based on 142 practices.

Subjects: Subjects included in a given year were those aged over 35 and registered on Dec 31 who had been continually registered with a practice for 6 months. Subjects with IHD were those with a preexisting READ code for angina, myocardial infarction or coronary revascularisation who were treated with a cardiovascular drug (BNF chapter 2) in that year. In 1998, the number of subjects with IHD was 33,911 .

Outcome measures: Record of a prescription for a LLD or a statin in a given year.

Exposure measures: We extracted age, sex, smoking status, time from diagnosis, IHD type, Region and the ACORN index. The ACORN index is an individual-level socio-economic measure, based on postcode-linkage with census data, prior to anonymisation and download of the data.

Results: In $19944.2 \%$ of men and $2.7 \%$ of women with IHD were prescribed statins. This rose to $55.0 \%$ and 39.9 by 2001 . Other LLDs were uncommon $(<3 \%$ in 2001). In $200132 \%$ of prescriptions for statins were for a possibly sub-optimal dose. The factors most strongly associated with statin prescription in 1998 were lower age, short time since diagnosis and type of IHD, (revascularisation $>$ Ml>angina). Men were more likely to receive statins than women $\mid O R=1.6295 \% \mathrm{Cl}$ (1.54-1.71)); this difference disappeared when adjusted for the other variables (adjusted $O R=1.06$ ) and appeared to be due to greater age and angina prevalence in women. Region had little effect but people in "Thriving" areas were slightly more likely to receive therapy.

Conclusions: LLD prescribing for subjects with IHD has increased markedly but remains below guideline targets with many patients on sub-optimal doses of statins. Many of these sub-optimal doses may be due to ignorance of the relative potency of the different statins. Sex differences in statin prescribing appear due to the higher prevalence of the "angina" diagnostic code in women with IHD.

\section{EXTENT OF SECONDARY PREVENTION OF CORONARY HEART DISEASE IN OLDER WOMEN AND MEN: THE CHALLENGE FOR THE NATIONAL SERVICE FRAMEWORK}

P.H. Whincup', D.A. Lawlor', J.R. Emberson'3 , K. Rees², S. Ebrahim', M. Walker'. 'Department of Public Health Sciences, St George's Hospital Medical School; ${ }^{2}$ Department of Social Medicine, University of Bristol; ${ }^{3}$ Department of Primary Care and Population Sciences, Royal Free UCL Medical School

Background: The importance of secondary prevention for patients with established coronary heart disease (CHD) is increasingly recognized, most recently in the National Service Framework for Coronary Heart Disease (NSF-CHD). We have examined the extent of effective risk factor control (as defined by the NSF-CHD) in the period immediately before implementation of the National Service Framework in two studies examining older British men and women aged 60-79 years.
Design: Cross sectional surveys in 24 towns across England, Wales and Scotland in women (British Women's Heart and Health Study) and in men (British Regional Heart Study) carried out between 1998 and 2001.

Participants: 3994 women and 4252 men, of whom 483 women $(12 \%)$ and 790 men (19\%) have evidence of a coronary heart disease (CHD) diagnosis in their General Practice record.

Main outcome measures: Prevalence $(95 \% \mathrm{Cl})$ of subjects with $\mathrm{CHD}$ requiring lifestyle modification and/or medication according to current guidelines.

Results: Among subjects with established CHD, 99\% (98 to 100\%) of women and $97 \%$ (96 to $98 \%$ ) of men had at least one risk factor that required modification. The prevalence of elevated blood pressure (SBP $140 \mathrm{mmHg}+$ or DBP $85 \mathrm{mmHg}+$ ) was $56 \%(51$ to $60 \%$ ) in women and $68 \%(65$ to $71 \%)$ in men. The prevalence of elevated total cholesterol level (5 mmol/L or more) was $87 \%$ (84 to 90 ) in women and $76 \%(73$ to $79 \%)$ in men. Prevalence of cigarette smoking was $10 \%(8$ to $13 \%)$ for women and $11 \%(9$ to $14 \%)$ for men. $74 \%(70$ to $78 \%$ ) of women and $73 \%$ (69 to $76 \%$ ) of men had a body mass index $>25 \mathrm{~kg} / \mathrm{m}^{2}$. Among women, $46 \%$ (42 to $51 \%$ ) were taking regular aspirin compared with $72 \%$ (69 to $75 \%$ ) in men; corresponding figures for statin use were $27 \%$ (23 to $31 \%)$ in women and $25 \%$ (22 to $28 \%$ ) in men. However, even among those subjects taking statins, a relatively low proportion had attained the target cholesterol level $(<5$ $\mathrm{mmol} / \mathrm{L})-32 \%$ (24 to $41 \%$ ) in women and $46 \%$ (38 to $53 \%$ ) in men.

Conclusion: There is considerable scope for improvement of secondary prevention of CHD in older British women and men in primary care. Efforts to improve secondary prevention should take precedence over increasing the role of primary care in the primary prevention of CHD.

\section{HSR II}

\section{THE IMPACT OF A NHS WALK-IN CENTRE ON LOCAL PRIMARY HEALTH CARE SERVICES}

R.T. Hsu, P.C. Lambert, M. Dixon-Woods, J.J. Kurinczuk. Department of Epidemiology \& Public Health, University of Leicester, UK

Objectives: To assess the impact of an NHS primary care walk-in centre on local primary and emergency health care services in order to investigate concerns that NHS walk-in centres might increase demand on those services.

Design: A prospective before-after comparative observational study.

Setting: Two towns in Leicestershire, one of which had an NHS walk-in centre from 1 July 2000 onwards.

Participants: Populations registered with participating GP practices.

Main outcome measures: The mean daily rate of GP practice emergency consultations, mean number of half-days to the sixth bookable GP routine appointment, mean daily rate of GP out-of-hours attendances, and attendance rates at local minor injuries units and A\&E departments.

Results: When the changes in the activity levels of the intervention population, from the six months before the NHS walk-in centre to the same six months after its introduction, were adjusted for the differences that occurred in the control population, there was no statistically significant difference in the daily rate of GP practice emergency consultations (mean difference $=-0.02$ per 1,000 population; $95 \%$ Cl: -0.75 to +0.71$)$, the time to the sixth bookable GP routine appointment (mean difference $=-0.24$ half-days; $95 \% \mathrm{Cl}:-1.85$ to +1.37 ), and the daily rate of attendances at GP out-of-hours services (mean difference $=+0.07$ per 1,000 population; $95 \% \mathrm{Cl}$ : -0.06 to $+0.19)$. Using Poisson random effects models adjusting for changes in the control population's attendance rates from before to after the NHS walk-in centre, the intervention population's attendance rate at the local minor injuries unit increased by $22 \%$ (rate ratio: $1.22 ; 95 \% \mathrm{Cl}$ : 1.12 to 1.33 ) and by $17 \%$ for non-ambulance attendances at local A\&E departments (rate ratio: $1.17 ; 95 \% \mathrm{Cl}: 1.03$ to 1.33 ).

Conclusions: The NHS walk-in centre did not significantly affect the workload of local GPs in their emergency surgeries, routine surgeries or out-of-hours service. However, there were significant differences in the changes in attendance rates at local minor injuries units and A\&E departments between the intervention and control populations. While the increase in attendances at the local minor injuries unit could be explained by its co-location with the NHS walk-in centre, the reasons for the findings relating to the non-ambulance attendances at the local $A \& E$ departments are more complex requiring further investigation. 


\section{QUESTIONNAIRE SURVEY OF USERS OF NHS WALK-IN CENTRES: OBSERVATIONAL STUDY}

C. Salisbury', T. Manku-Scott', L. Moore'2, M. Chalder', D. Sharp' IDivision of Primary Health Care, University of Bristol; ${ }^{2}$ School of Social Sciences, Cardiff University

Background: Forty NHS walk-in centres have been established as part of the government's commitment to modernise health care, representing an investment of $£ 280 \mathrm{M}$ over 3 years. These centres are led by nurses, supported by clinical assessment software. They are intended to provide quick and convenient access to high quality care, helping people to manage problems themselves and relieving pressure on other health service providers.

Objectives: To determine characteristics and experiences of people consulting NHS walk-in centres compared with general practice

Design: Observational study using questionnaires

Setting: 38 walk-in centres and 34 neighbouring general practices

Participants: 3856 people attending walk-in centres and 2373 people attending general practices on a 'same-day' basis.

Main outcome measures: Socio-demographic characteristics, reasons for consulting, attitudes to continuity, satisfaction, enablement, referrals, intentions.

Results: Walk-in centre visitors were more likely to be owner-occupiers $(55 \% \vee 49 \% ; P<0.001)$ to have further education $(25 \% \vee 19 \% ; P=0.006)$ and to be white $(88 \% \vee 84 \% ; P<0.001)$ than general practice visitors. Main reasons for attending a walk-in centre were speed of access and convenience. Walk-in centre visitors were more likely to attend on the first day of illness $(28 \%$ v. $10 \% ; P<0.001)$, less likely to expect a prescription $(38 \% \vee 70 \%, P<0.001)$, and placed less importance on continuity of care (adjusted odds ratio $0.58 ; 0.50$ to 0.68 ) than general practice visitors. People were more satisfied with walk-in centres (adjusted mean difference $6.6 \% ; 5.0$ to $8.2 \%$ ). Enablement scores were slightly higher in general practice (adjusted mean difference $3.2 ; 0.1$ to 5.4 ). Following the consultation $13 \%$ of walk-in centre visitors were referred to general practice, but $32 \%$ intended to make an appointment.

Conclusions: NHS walk-in centres improve access to care, but not necessarily for those people with greatest health needs. People predominantly attend with problems of recent onset as an alternative to existing health providers, and are very satisfied with the care received. These benefits need to be considered in relation to the cost, and in comparison with other ways of improving access to health care.

\section{COMMISSIONING IN THE NHS: A QUALITATIVE ANALYSIS OF PRIMARY CARE TRUSTS AND NHS TRUSTS}

K. Baxter', M. Weiss', J Le Grand'. 'Division of Primary Health Care, University of Bristol; ${ }^{2}$ Department of Social Policy, London School of Economics and Political Science

Objective: To determine the value of principal-agent theory in the analysis of the commissioning process between Primary Care Trusts (PCTs) and NHS Trusts. Principal-agent theory considers problems around delegated decisions: that is, an individual (the agent) making decisions that are in the interests of another (the principal) in return for some form of payment. This study considers the delivery of secondary care by NHS Trusts (as agents) for PCTs (as principals).

Setting: Three PCTs and their local acute NHS Trusts in the English NHS

Design: Observation and qualitative analysis of meetings. Each of the three study PCTs was involved in a series of meetings with their local acute NHS Trust, their Health Authority and neighbouring PCTs, either together or individually. The meetings were held between November 2001 and March 2002 and their purpose was to agree Service and Financial Frameworks (SaFFs) for 2002/2003. Qualitative data analysis software was used to assist analysis of written notes of the meetings.

Participants: PCT, acute NHS Trust and Health Authority managers and clinicians.

Main outcome measures: A model of relationships between PCTs and acute NHS Trusts.

Results: The theory assumes that principals (PCTs) and agents (NHS Trusts) have different objectives. This is not clear-cut for PCTs and NHS Trusts; both have financial and other (e.g. access) targets to meet, some shared, others not. The theory also assumes that agents are not involved in devising the payment schedules offered to them by their principals. In the NHS this is not the case. NHS Trusts are engaged actively in PCTs' discussions about appropriate levels of service and its costs. In addition, the context within which PCTs and NHS Trusts are working plays an important role in determining the shape of these relationships, for example, the financial deficits of NHS Trusts dominate some discussions. The principal agent relationship may be reversed, with PCTs acting as agents for NHS Trusts that are seeking to meet their own agendas.

Conclusions: PCTs are shaping up to be weak purchasers. The government should be careful if it plans to rely on PCTs as instruments of quality improvement and efficiency in the secondary care sector.

\section{NON-PRACTISING NURSES IN WALES: WHO ARE THEY AND WHAT MIGHT BRING THEM BACK TO THE NHS?}

S. Drayton 1, H. Snooks², A. Williams ${ }^{3}$, J. Williams², A. Evered'4. 'lechyd Morgannwg Health Authority; ${ }^{2}$ Centre for Postgraduate Studies, Clinical School University of Wales Swansea; ${ }^{3}$ School of Health Science, University of Wales Swansea; ${ }^{4}$ Bro Morgannwg NHS Trust

Background: There is currently a shortage of nurses and midwives in Wales, as elsewhere in the UK. Although some research has been undertaken to understand reasons for nurses leaving the NHS, little is known about those who have ceased to practise and let their UKCC registration lapse.

Objectives: To: 1. Identify the number of nurses and midwives who have let their UKCC registration lapse in Wales. 2. Identify reasons given by these ex-nurses and midwives for leaving the NHS - and factors that might bring them back.

Participants: Nurses and midwives who have let their UKCC registration lapse in Wales, and in particular in lechyd Morgannwg Health Authority.

Study design: Analysis of routine UKCC data. Postal survey of ex-nurses in IMH HA, with questionnaire design informed by literature review and focus group.

Results: There are currently 30,521 practitioners in Wales holding effective UKCC registration. In addition, 8,087 nurses across Wales have let their registration lapse in the last 15 years, $55 \%$ of these aged less than 55 .

Of 1,397 ex-nurses who were sent questionnaires, 573 returned them. Females were more likely to respond $(42 \%$ vs $31 \%, p=.02)$ as were those aged over $50(46 \%$ vs $30 \%, p<.01)$.

The main reasons given for leaving were: ill health $(n=254)$; retirement $(\mathrm{n}=150)$; child care $(\mathrm{n}=81)$; stressful environment $(n=80)$ and career opportunities $(n=44)$. Overall $16.2 \%(n=93)$ stated that they intended to return to practice, although this was higher in the younger age group $<55: 40.0 \% ; p<.001)$. The factors most often cited as likely to make a big difference to their returning to work were: reduction in staff shortage $(83.5 \%)$; better advice $(82.5 \%)$; more patient contact $(79.5 \%)$; better choice of day/night shift $(78.8 \%)$; more hands-on practice $(76.8 \%)$; more support during 'return to work' $(75.0 \%)$; shorter shifts $(70.9 \%)$; and later start $(68.4 \%)$. If issues raised were resolved, $87.1 \%$ of those stating that they intended to return to work said that nothing else would stop them from returning.

Conclusions: A high proportion of nurses appear to be leaving the NHS due to ill health. However, there is also a pool of trained nurses and midwives who have stopped practising for reasons which may be resolvable, with increased flexibility on the part of employers. This may be more cost-effective than some of the more radical measures recently taken by some Trusts.

\section{WRITING TO PATIENTS: IMPACT ON PATIENT RECALL AND SATISFACTION}

M. O' Reilly', M. Cahill', I.J. Perry ${ }^{3}$ ' 'Department of Epidemiology \& Public Health, University College Cork; ${ }^{2}$ Haematology Department, Mid-Western Regional Hospital; ${ }^{3}$ Department of Epidemiology \& Public Health, University College Cork

Background: The NHS Plan has made a commitment to copying clinicians' letters to patients as of right and Your Guide to the NHS also states that, in future, this will happen routinely. Providing patients with a detailed letter summarising their outpatient consultation has been highlighted in recent studies to be of potential value to patients.

Objective: To evaluate the feasibility, acceptability and effectiveness of a summary letter from a consultant to new outpatients attending a haematology outpatient clinic in a general hospital setting. This summary letter is copied to the referring medical professional and replaces the current standard clinic letter. 
Design: A randomised controlled trial involving 150 referrals to haematology outpatients, of whom 77 were randomised to receive a letter summarising the consultation, with a copy to their GP and 73 controls who received a note thanking them for attending the clinic with a standard letter to their GP. Intervention and control patients were assessed for recall (cued recall method) and satisfaction using a standardised structured interview. Patient recall was compared with observation notes of the consultation to indicate a recall score. 13 patients did not attend for their follow-up appointment. 102 referring medical professionals were also surveyed to seek their opinions of the use of summary letters.

Results: 71 intervention and 66 control patients returned for their second visit to outpatients. Strongly positive views on the summary letter were expressed by intervention patients, reflecting high satisfaction rates. $80 \%$ were very pleased or pleased to receive a summary letter and $81 \%$ found the letter to be very useful or useful. Intervention patients recalled marginally (but not significantly) more information than control patients, recalling on average $63 \%$ of the information provided to them versus $60 \%$ of information for controls. The consultant estimated that patient summary letters took an average of 6.8 minutes to dictate compared with 3-4 minutes for a new patient standard clinic letter. 92\% ( $n=59)$ of general practitioners and consultants rated the patient summary letter as a 'very useful' or 'useful' method of doctor-patient communications, just $8 \%$ rated the letter as 'useless'

Conclusions: Patients are overwhelmingly in favour of patient summary letters, expressing strongly positive views. The main impact of summary letters is on patients reported satisfaction. There was no observable impact on patient recall. The practice of consultants sending a patient summary letter is feasible and enhances patient experiences and satisfaction with the consultation.

\section{Health of older people}

\section{WHY DO OLDER PEOPLE OPT-OUT OF RESEARCH STUDIES?}

I.K. Crombie', A.R. McGinnis', L. Irvine', M.E.T. McMurdo², B. Williams', P.W. Slane', E.M. Alder'. 'Departments of Epidemiology and Public Health; ${ }^{2}$ Medicine, University of Dundee; ${ }^{3}$ Wallacetown Health Centre, Dundee; ${ }^{4}$ Department of Health and Life Sciences, Napier University, Edinburgh

Background: Low rates of participation are common in research studies among older people. As part of a study on physical activity among older people we explored factors influencing refusal to participate and the reasons older people give for non-participation.

Methods: The study was conducted in two phases. Subjects were initially approached by their GP and were given the opportunity to opt out by returning a postcard to the GP practice. Phase 1 investigated the effect of socio-demographic factors on participation rates. Phase 2 explored the reasons for non-participation. Subjects who opted out were approached by their GP and asked to complete a short questionnaire on reasons for non-participation.

Results: Phase 1: Overall, $54 \%$ of those invited to take part subsequently opted out. Opting out of the study was influenced by age, gender and social class. The highest refusal rate was among the eldest people living in the most deprived areas. While $29 \%$ of younger men (65-74 years) from areas of low deprivation refused to take part, $60 \%$ of older men (75-84 years) from areas of high deprivation refused. Refusal was even higher among women. Half of the younger women from areas of low deprivation refused while $65 \%$ of older women from the most deprived areas refused.

Phase 2: Questionnaires have been distributed to those who opted out of the study. So far a response rate of $64 \%$ has been achieved. Possible factors for non-participation being explored are whether older people feel that research is not relevant to them, because they think they are too old or not well enough to contribute. Other possible reasons include lack of time, whether an interview at home would be too intrusive or whether the topic is not of interest.

Conclusions: The findings from Phase 1 show that the response to the invitation to take part is strongly socially patterned. Older people, especially women, from more deprived areas are unwilling to participate in this type of research. Phase 2 will clarify the reasons for this reluctance.

Funding: The Chief Scientist Office, Scottish Executive Health Department.

\section{NURSE-LED CASE MANAGEMENT OF OLDER PATIENTS IN GENERAL PRACTICE: AN EVALUATION}

J. Townsend', J. Hodgkinson', M. McKinnon', R. McGurk', I. Machen', A. Oates' ${ }^{2}$ J. Adolph ${ }^{3}$, J. Machen'. 'Centre for Research in Primary and Community Care, University of Hertfordshire, Hatfield; ${ }^{2} G P ;{ }^{3}$ Practice Nurse

Background: Case management has been proposed as an effective way of improving the standards of care, quality of life and functional independence enjoyed by older adults, by reducing the fragmentation of care and by providing assessment and monitoring functions, to enable older adults to remain in the community for longer and reducing long-term costs to the health service.

Objective: To evaluate the effectiveness of nurse-led case management of older adults in primary care in improving quality of life, health status and satisfaction with practice and cost effectiveness.

Design: Randomised controlled trial of nurse-led case management against usual care.

Setting: Ten general practices in Hertfordshire and London.

Participants: 4423 patients over 75 years living in the community.

Intervention: Following appropriate training, a GP, Practice and District Nurses and Social Service worker met regularly to agree care pathways and undertake regular review of care for 18 months, using 96 specially selected and adapted evidence-based guidelines.

Main outcome measures: Surgery consultations, home visits, nursing home admissions, mortality rates, numbers and lengths of hospital stay from practice records and health authorities. Patient perceived quality of life and satisfaction with care assessed by qualitative interviews and questionnaires (baseline, mid-point, end-point), incorporating EQ5D and Geriatric Depression Scales. Participating health service staff were interviewed about their perspectives of case management. Cost effectiveness was assessed.

Results: Self-perceived health state measured by EQ5D improved significantly for patients in the intervention practices; nursing home admissions were lower for intervention patients in the follow-up period lodds ratio. Control group surgery attendance increased between baseline and follow-up more than in the intervention group; home visits increased more for intervention patients. No differences were found for hospital admissions, depression or mortality. Interviews with involved health service staff revealed enthusiasm for their experience of case management, especially its positive impact on closer working relationships. Costs to the health service were marginally reduced overall.

Conclusion: Nurse-led case management was associated with a positive impact on patient self-perceived health, affected the number and type of contacts with health care professionals, and reduced the likelihood of nursing home admission, without increasing expenditure for the health service.

\section{SOCIAL ACTIVITY AND LONG-TERM MORTALITY AMONG OLDER PEOPLE}

P.A. Bath, A. Gardiner. Department of Information Studies, University of Sheffield, Sheffield, UK

Background: Active social engagement is an important component of successful ageing. Although some research has demonstrated that higher levels of social activity improve survival among older people, it remains unclear whether social activity is an independent risk factor for mortality in older men and women. The aim of this study was to examine the relationship between social activity and long-term mortality in older men and women.

Methods: A representative sample of 1042 community-dwelling people (406 men; 636 women) aged 65 and over was interviewed in 1985 in the Nottingham Longitudinal Study of Activity and Ageing. Social activity, physical health and psychological well-being were assessed using validated health indices. Customary physical activity was assessed by recording activities contributing to stamina, muscle strength and joint flexibility. Information on mortality within the sample was provided by the UK NHS Central Register to 29th February 2000. Respondents were divided into 3 activity groupings according to tertile ranges for the social activity scores (high, intermediate and low). A series of Cox regression models was used to determine whether level of social engagement predicted 15-year mortality for men and women separately.

Results: In unadjusted models, there was increased mortality among men with medium (Hazard ratio $(\mathrm{HR})=1.59$; $95 \% \mathrm{Cl}=1.16,2.18 ; p<0.01)$ and low $(\mathrm{HR}=2.23 ; 95 \% \mathrm{Cl}=1.62,3.07$; $p<0.0001)$ levels of social activity and among women with medium $(\mathrm{HR}=1.49 ; 95 \% \mathrm{Cl}=1.13,1.96 ; \quad p<0.01)$ and low $(\mathrm{HR}=2.56$; $95 \% \mathrm{Cl}=1.93,3.39 ; p<0.0001)$ levels, relative to men/women with 
high levels of activity. When adjusting for demographics, physical health and psychological well-being, men with medium $(H R=1.40$; $95 \% \mathrm{Cl}=1.01,1.94 ; p<0.05)$ and low $(\mathrm{HR}=1.59 ; 95 \% \mathrm{Cl}=1.08,2.33$; $p<0.05$ ) levels of social activity still showed increased mortality, but not when physical activity was included in the model. When adjusting for demographics and physical health among women, medium level of social activity was not predictive of mortality, although women with low $(\mathrm{HR}=1.69 ; 95 \% \mathrm{Cl}=1.23,2.31 ; p<0.001)$ levels of social activity still showed increased mortality. When adjusting for demographics, physical health and psychological well-being, women with low $(\mathrm{HR}=1.61 ; 95 \% \mathrm{Cl}=1.16,2.24 ; p<0.01)$ levels of social activity still showed increased mortality, but not when physical activity was included in the model.

Conclusions: Older men and women engaging in lower levels of social activity had increased mortality. The improved survival shown by older people engaging in high levels of social activity was explained by demographic factors, physical health, psychological well-being and physical activity. Promoting social activity may help improve these components of healthy ageing and improve long-term health outcomes in older men and women.

\section{THE INCIDENCE OF DEMENTIA IN ENGLAND AND WALES}

MRC CFAS', F.E. Matthews'², C. Brayne 3 , C. McCracken ${ }^{4}{ }^{1} M R C$ Cognitive function and ageing study (www.mrc-bsu.cam.ac.uk/cfas); ${ }^{2}$ MRC Biostatistics Unit, Institute of Public Health, Cambridge; ${ }^{3}$ Department of Public Health and Primary Care, Institute of Public Health, Cambridge; ${ }^{4}$ Department of Psychiatry, University of Liverpool

Purpose: To estimate the incidence of dementia in a population based study in England and Wales.

Methods: The Medical Research Council cognitive function and ageing study (MRC CFAS) is a multi-centre two-phase two-stage population based cohort study. One of its primary aims was the estimation of the incidence of dementia within a community setting. 13,004 individuals from five centres across England and Wales (Cambridgeshire, Gwynedd - Wales, Newcastle-upon-tyne, Nottingham and Oxford) participated in interviews two years apart. At each wave more detailed interviews were undertaken of cognitively frail individuals and a random sample of all others, stratified by age 65-74 years and 75 and over. The aim was to sample 2,500 individuals from each centre, and obtain more detailed information (including AGECAT organicity diagnosis) from 500 of these.

Results: Incidence of dementia by age shows the expected increase in rates, with men's rates slightly lower than women's rates. The rates increase almost exponentially from less than 10 per 1000 person-years per year in the youngest ages (65-69) to more than 100 in the oldest old ( 90 and above). The ten- fold increase seen is similar in both men and women. Some systematic variation is seen in rates between the centres.

Conclusion: Incidence of dementia is a substantial community problem within the oldest of our society despite their high mortality. The long term effects of the exponential increase will have increasing importance as the very oldest ages become commonplace within society.

\section{A CONCEPTUAL FRAMEWORK FOR HEALTH-RELATED QUALITY OF LIFE IN PEOPLE WITH DEMENTIA}

R.H. Harwood', S.C. Smith², J. Murray 3 , B. Foley', J. Cook ${ }^{3}$, D.L. Lamping², S. Banerjee'. 'Queen's Medical Centre, Nottingham; 'London School of Hygiene and Tropical Medicine; ${ }^{3}$ Institute of Psychiatry, London

Background: An assessment of the validity of a health outcome measure requires a clear definition and description of the construct that is to be measured. These may vary according to the disease being studied. Measuring a subjective phenomenon such as health-related quality of life (HRQL) is acknowledged to be difficult. There are also difficulties in obtaining reliable self-reports from people with dementia. Clear definition of the construct to be measured is therefore all the more important. The few existing studies that have attempted to define HRQL conceptually for people with dementia have concluded that HRQL in dementia is shaped by the disease, and that there are domains of $H R Q L$ that are unique to dementia.

Methods: We combined top-down (from existing literature) and bottom-up (new qualitative data) approaches to develop a diseasespecific conceptual framework of HRQL for people with dementia. In-depth semi-structured qualitative interviews were conducted with 19 people with dementia and 20 informal carers.

Findings: Content analysis revealed 5 domains: daily activities and looking after yourself (e.g. getting the things you need from the shops), health and well-being (e.g. happiness/ depression), cognitive functioning (e.g. making your mind up), social relationships (e.g. companionship) and self-concept (e.g. feeling useful). All of these domains were cited by both people with dementia and their carers, but closer consideration of the qualitative data indicated that people with dementia tended to adopt a positive view and focused on how they felt at the time of the interview. By contrast, carers were generally less positive and presented a more longitudinal perspective, often describing how things had changed for the worse over time.

Conclusion: The conceptual framework helps us understand what makes up good and bad quality of life in dementia. This provides the opportunity to develop new interventions to improve quality of life, and also provides a basis for evaluating such interventions, including drugs, other health care, social and psychological therapies. The conceptual framework forms the basis for a new measure of HRQL for people with dementia (DEMQOL). Final field testing for validity and reliability of this new measure is in progress.

\section{Early life risk factors}

\section{LOW BIRTH WEIGHT AND BLOOD PRESSURE IN ADOLESCENCE AND EARLY ADULTHOOD}

P.O.D. Pharoah, C.J. Stevenson, C.R. West. Department of Public Health, University of Liverpool, Liverpool

Background: It has been hypothesised that raised adult blood pressure (BP) has its origin in environmental factors acting during early fetal development. The hypothesis has considerable public health implications if prevention of hypertension is a major goal. The association of low birth weight with BP in adolescence and early adulthood was examined.

Methods: A cohort of infants of birth weight $<=1500 \mathrm{~g}$ (VLBW) born to mothers resident in the County of Merseyside was obtained from the birth notifications and a comparison group individually matched for age, sex and school were followed in adolescence and early adulthood. The BP, height and weight were measured when the children aged 15 years and BP measured again when they were aged 20 years. The birth weight ratio (actual birth weight/expected birth weight for gestational age) was used as a measure of intrauterine growth. Twins and children with clinical disability were excluded from the analysis.

Results: There were 128 singleton children free of clinical disability and attending mainstream schools with their matched controls. At age 15 years, the index cases were significantly shorter, lighter in weight and had a higher systolic BP than the matched comparison group (systolic BP difference $5.3 \mathrm{~mm}$, after adjusting for height and weight; $\mathrm{p}<0.001$ ). The adjusted systolic BP difference persisted at age 20 years $(6.5 \mathrm{~mm} ; \mathrm{p}<0.001)$. The diastolic BP difference was not significant. No association between birth weight ratio and BP was found. There was highly significant $6.0 \mathrm{~mm}$ increase in and correlation between systolic BP between the ages of 15 and $20(r=$ $0.47 ; p<0.001)$ indicating that there is tracking of systolic BP from adolescence to adulthood.

Conclusions: There is a significant increase in systolic BP associated VLBW. This is more attributable to being born pre-term rather than to growth restriction in utero. There is tracking of BP between adolescence and adulthood.

\section{INFLUENCE OF SIZE AT BIRTH ON THE CARDIOVASCULAR RISK PROFILE OF CONTEMPORARY BRITISH ADOLESCENTS}

C.G. Owen, P.H. Whincup, K. Odoki, J.A. Gilg, D.G. Cook. Department of Public Health Sciences, St George's Hospital Medical School, Cranmer Terrace, London, SWI7 ORE

Objectives: Low birthweight, as a measure of poor fetal nutrition, is implicated as a marker of increased cardiovascular risk. We have examined the influence of birthweight on the cardiovascular risk profile of adolescents (blood pressure, serum cholesterol, and fasting insulin) and compared the strength of associations with those of current adiposity.

Design: Cross sectional survey of adolescents, with retrospective ascertainment of birthweight from birth records or parental recall.

Setting: 69 schools from 10 English and Welsh towns.

Main outcome measures: Systolic blood pressure (SBP), serum total cholesterol (TC) and fasting insulin. All analyses adjusted for town, age, current size (height and BMI) and pubertal status.

Participants: School children (53\% male) aged between 12.7 and 16.4 years (mean 14.8 years) with measurement of SBP $(\mathrm{N}=2643)$, TC $(\mathrm{N}=1532)$, and fasting insulin $(\mathrm{N}=1369)$. 
Results: The association between birthweight and cardiovascular risk appeared to differ between the sexes. In girls, birthweight was inversely related to SBP $(-3.10 \mathrm{mmHg} / \mathrm{kg}, 95 \% \mathrm{Cl}-4.48$ to -1.72$)$ and insulin $(-7.2 \% / \mathrm{kg}, 95 \% \mathrm{Cl}-13.0$ to -0.9$)$. In boys, associations appeared weaker both for SBP $(-1.10 \mathrm{mmHg} / \mathrm{kg}, 95 \% \mathrm{Cl}-2.39$ to $0.20)$ and insulin $(-1.0 \% / \mathrm{kg}, 95 \% \mathrm{Cl}-7.0$ to $5.6 \%)$; there was evidence of a sex interaction for SBP $(P=0.05)$, although not for insulin $(P=0.15)$. There was no strong association between birthweight and TC for either sex. Current adiposity showed strong positive associations with all three cardiovascular risk markers. Even among girls (who had stronger birthweight associations with cardiovascular risk) the effects of an inter-quartile range increase in BMI were substantially greater than those for an equivalent change in birthweight ( 3 times greater for SBP, 4 times for insulin, and 3 times for TC).

Conclusion: Though fetal nutrition may be related to the cardiovascular risk profile of contemporary adolescents (particularly in girls), current adiposity is a more important influence. Control of adiposity in young people is likely to be a more important priority for cardiovascular prevention than improvement of fetal nutrition.

\section{BIRTH WEIGHT, CHILDHOOD BODY SIZE AND ADULT MUSCLE STRENGTH: A LIFE COURSE STUDY}

D. Kuh', E.J. Bassey², R. Hardy', A. Aihie Sayer ${ }^{3}$, M.E.J. Wadsworth', C. Cooper'. 'Medical Research Council National Survey of Health \& Development, Royal Free \& University College, London; ${ }^{2}$ School of Biomedical Sciences, Queens Medical Centre, Nottingham University; ${ }^{3}$ Medical Research Council Environmental Epidemiology Unit, Southampton University

Objectives: Muscle strength is important in maintaining physical performance into old age. It is hypothesised that environmental influences during gestation may have long term effects on adult muscle strength. We examined the effect of birth weight on hand grip strength in middle age, and whether the effect was modified by later body size or confounded by lifetime social class.

Methods: Prospective national birth cohort of 1371 men and 1404 women aged 53 years, with measures of childhood and current body size and social class. Tests of interaction were conducted in the fully adjusted regression models to assess whether the effects of birth weight varied by childhood or current body size.

Results: Among both men and women, grip strength increased progressively with increasing weight at birth, 7 and 53 years; and with increasing height at 7 and 53 years $(p<0.001$ for all relationships with the exception of grip strength and adult weight among women). After adjusting for childhood and adult body size, the estimated mean increase in grip strength (95\% confidence interval) per $\mathrm{kg}$ increase in birth weight was $1.9 \mathrm{~kg}(0.52-3.2 \mathrm{~kg})$ for men and $1.2 \mathrm{~kg}(0.29-2.1 \mathrm{~kg})$ for women. Adult social class was positively associated with grip strength, but the relationship with childhood social class was weaker and not statistically significant. An effect of birth weight on grip strength remained after adjusting for childhood and current body size and social class, and did not vary by later body size.

Conclusions: This study is the first to show that birth weight has an important influence on muscle strength in midlife independent of later body size and social class. It suggests that birth weight is related to an individual's number of muscle fibres which are established by birth and that even in middle age compensating hypertrophy may be inadequate. As the inevitable loss of muscle fibres proceeds in old age, a deficit in the number of fibres could threaten quality of life and independence. There are health care implications for an increasingly aged society.

\section{BIRTHWEIGHT, CHILDHOOD SOCIO-ECONOMIC ENVIRONMENT AND COGNITIVE DEVELOPMENT IN THE 1958 BRITISH BIRTH COHORT}

B.J.M.H. Jefferis', C. Power', C. Hertzman'2. 'Institute of Child Health, Centre for Paediatric Epidemiology and Biostatistics, 30 Guilford Street, London, WCIN 1EH, U.K; ${ }^{2}$ Department of Health Care and Epidemiology, Faculty of Medicine, University of British Columbia, Vancouver, B.C. V6T 1Z3, Canada

Objectives: To examine (i) the combined effect of social class and weight at birth on cognitive trajectories during school age (ii) associations between birthweight and educational outcomes through to age 33 years.

Design: Birth cohort consisting of all births in England, Scotland and Wales 3-9th March 1958.

Participants: 10,845 singleton males and females with information on birthweight, social class and cognitive tests.
Main outcome measures: Reading, maths, draw-a-man, copying designs, verbal and non-verbal ability tests at ages 7,11 and 16 years, highest educational qualifications achieved by 33 years, trajectories of maths standardised scores 7-16 years.

Results: All childhood cognitive tests and educational achievements increased significantly with increasing birth weight. Exclusion of children with disability or premature birth (less than 37 weeks gestation) did not change the relationships. Analysis of maths scores at 7 years and of highest qualifications achieved by 33 years showed that the relationships were robust to adjustment for potential confounding factors, (gestational age, maternal age, social class at birth, infant feeding, parity and parental education). For each kilogram increase in birthweight maths $z$-score increased by 0.17 (adjusted estimate $0.15,95 \% \mathrm{Cl} 0.10,0.21$ ) for males and 0.21 (adjusted estimate $0.20,95 \% \mathrm{Cl} 0.14,0.25$ ) for females. Trajectories of maths $z$-scores between 7 and 16 years diverge for different social class groups: subjects from classes I\&ll increased their relative position on the score with increasing age, whilst classes IV\&V showed a relative decline with increasing age. In contrast, the effect of birthweight did not change over time. Birthweight explained much less of the variation in cognition than social class (range $0.5-1.5 \%$, compared to 2.9$12.5 \%$ ).

Conclusions: The postnatal environment has an overwhelming influence on cognitive function through to early adulthood, but these strong effects do not explain the weaker but independent association with birthweight.

\section{WHAT EFFECT HAS LOW BIRTH WEIGHT ON SCHOOL EDUCATIONAL ATTAINMENT?}

C.J. Stevenson, C.R. West, P.O.D. Pharoah. Department of Public Health, University of Liverpool, Liverpool

Background: Infants of low birth weight compared to those of normal birth weight are at significantly greater risk of disability, in particular cerebral palsy. Longer term follow up has shown that children who were of low birth weight also perform less well on measures of cognitive, motor and psychological function. We report here a comparison of GCSE ' $O$ ' level results in children of low birth weight and their matched controls.

Methods: A cohort of infants of birth weight $<=1500 \mathrm{~g}$ born to mothers resident in the County of Merseyside was obtained from the birth notifications. When aged 8 years, a comparison group individually matched for age, sex and school were obtained and all were assessed using measures of cognitive, motor and psychological function. When aged 15 years, their GCSE ' $O$ ' level results were obtained. A numerical score was given to each grade of ' $O$ ' level ranging from 8 for $A^{*}, 7$ for $A$ down to 1 for Grade $G$. Children attending 'special' schools and those with clinical disability were excluded from the analysis.

Results: There were 167 children free of clinical disability and attending mainstream schools with their matched controls. GCSE results were obtained for $138(83 \%)$ pairs.

The mean total points score was 32.4 in the index group and 36.5 in the comparison group (Difference between means 4.1 (95\% Cl 0.6 to 7.7); $p=0.001$ ). The mean points score per GCSE subject was 3.8 in the index group and 4.2 in the comparison group (Difference between means 0.4 (95\% Cl 0.1 to 0.7$) ; p=0.01)$.

Conclusions: As index and comparison groups were matched for school and class in school, it is unlikely that the difference in performance is related to teaching. Schools tend to have socially homogenous catchment areas so that it is unlikely that postnatal social environmental factors account for the performance difference. The factors inherent in being born of low birth weight most likely to account for the differences observed.

\section{Cancer}

\section{DOES THE DISTANCE FROM PAEDIATRIC ONCOLOGY CENTRES AFFECT THE QUALITY OF LIFE OF FAMILIES WITH CHILDREN ON CANCER CHEMOTHERAPY?}

P. Kearney', M. O' Reilly2, A. Mc Ninch', T. Parkyn ${ }^{3}$, M. Mott ${ }^{4}$. 'Departments of Paediatrics and Child Health,' University College Cork; 2Epidemiology and Public Health, University College Cork; ${ }^{3}$ Paediatric Unit, Royal Devon and Exeter Hospital; ${ }^{4}$ Department of Paediatric Oncology, University of Bristol

Aims: The aims of this project were to explore, describe and understand the experience of travelling to paediatric oncology 
centres, and to identify the burdens of distance for the families of children with cancer and leukaemia.

Methods: This initial exploration of family experiences required a qualitative methodology. Two paediatric units associated with centres that had different policies of decentralising care cooperated with the study. Parents were purposefully selected and invited to participate. Four focus group interviews moderated by an experienced sociologist were recorded. A total of 22 parents ( 5 fathers and 17 mothers) were interviewed. The transcripts were analysed using a grounded theory approach.

Results: The experience of having a child with cancer or leukaemia was very intense for both groups, but there were differences related to the burden of travel. Travel was hardly an issue for the decentralised group. The parents using a more centralised service identified several burdens of travel. They were exhausted by the uncertainty of travel arrangements and the huge distances involved because of repetitive round trips. The experience of travelling with a sick child was frightening and aggravated by the worry of car accidents and the risk of infection in crowded trains. Some of the children's behaviour was barely tolerable during travel. Serious financial problems resulted from the need for a second car, accommodation near the centre, and time lost from work. Family disruption was more severe for the families associated with the centralised service and most parents were very aware of sibling neglect.

Conclusions: The recommendation of one paediatric oncology centre per 5 million population remains suitable in dense population areas, where parents are within commuting distance. Maximal devolution of care reduces the burden of travel on families and provides a better solution than centralisation in less densely populated areas. There is a need for appropriate standards of staffing and accommodation for units with maximal devolution of care.

\section{NUMBER OF LYMPH NODES IDENTIFIED BY PATHOLOGISTS PREDICTS FIVE-YEAR SURVIVAL OF PATIENTS WITH COLORECTAL CANCER: OBSERVATIONAL STUDY OF 3,638 CASES}

S. George, J.N. Primrose, J.A.E. Smith, P. King, H. Wrigley, M. Mullee, R. Talbot, C. du Boulay. Wessex Colorectal Cancer Working Group, SouthWest Cancer Intelligence Service \& University of Southampton, UK

Introduction: It has been shown that survival in Dukes' B colorectal cancer patients is related to the number of lymph nodes examined by a pathologist, greater survival being associated with more nodes examined. Authors have generally concluded that this relationship is due to stage drift (i.e. that had more nodes been examined some of those staged as Dukes' B would have been found to be Dukes' C patients, with consequently poorer survival). We examine this hypothesis in a large cohort of colorectal patients of all Dukes' stages.

Methods: All patients with a new diagnosis of colorectal cancer made in the former Wessex Health Region were prospectively recorded between September 1991 and August 1994. Data were extracted from medical records and 5,176 cases of colorectal cancer identified. Details of presentation, diagnosis, staging and management were recorded and patients followed up for five years. We excluded from analysis cases where stage or data on number of nodes removed were absent. Stratification was into three groups by number of nodes identified. We made survival comparisons using KaplanMeier curves and the log rank test. We also undertook an analysis in a subset of 893 patients who had an indication from the examining pathologist of the degree of lymphocytic infiltration of the primary tumour.

Results: Our dataset consisted of 3,638 cases: 407 Dukes' A 1,473 Dukes' B, 964 Dukes' C and 794 Dukes' 'D'. Overall a survival advantage was shown in patients who had $>10$ nodes identified over those who had 5-10, and a similar advantage in patients who had $5-10$ over those who had $\leqslant 4(p<0.001)$. A survival advantage of similar magnitude was also observed within each stage, although these differences only achieved statistical significance in Dukes' B $(p=0.0039)$ and Dukes' $C(p=0.0160)$ cases. The difference remained after adjustment for age, and for receipt of chemotherapy using Cox regression. The overall pattern was repeated in the sub-group where data on lymphocytic infiltration were available. A significant survival advantage was also noted in those with prominent rather than mild lymphocytic infiltration $(p<0.00001)$ : the Mean (Median) number of nodes in the prominent $(n=299)$ and mild $(n=594)$ groups being $8.5(8)$ and 7.9 (7) respectively (MannWhitney test: $p=0.018)$. Pattern of lymph node yield was similar amongst individual surgeons and amongst pathologists.

Conclusions: Stage drift cannot explain these results as the survival advantage applies to the whole group. Tumours with a pronounced lymphocytic infiltrate have a good prognosis compared with those that have no such infiltration. It may be that reactive enlargement of lymph nodes in the mesentery makes nodes easier to find, reflects immune response to the tumour, and thus indirectly impacts upon survival.

\section{GEOGRAPHICAL INEQUALITIES IN LUNG CANCER MANAGEMENT AND SURVIVAL IN SOUTH EAST ENGLAND: EVIDENCE OF VARIATION IN ACCESS TO ONCOLOGY SERVICES?}

R. Jack', M. Gulliford', J. Ferguson'2, H. Møller ${ }^{3}$. 'Department of Public Health Sciences, King's College London; 'Lambeth Southwark and Lewisham Health Authority, London; ${ }^{3}$ Thames Cancer Registry, King's College London

Background: Survival of patients with lung cancer is lower in England than in Europe or North America.

Objective: We evaluated whether the management and survival of patients with lung cancer varied among 26 health authorities in South East England.

Setting: Twenty-six health authorities in South East England in 1995-1999.

Subjects: Subjects registered with lung cancer at the Thames Cancer Registry (ICD-10 codes C33-C34).

Main measures: Basis of diagnosis; use of surgery, chemotherapy and radiotherapy; and one- and three-year survival rates. Random effects models were used to estimate health authority level variances.

Results: The proportions of patients receiving active treatment varied among health authorities ranging from $11 \%$ to $28 \%$ for non-investigative surgery, $4 \%$ to $17 \%$ for any chemotherapy, $8 \%$ to $30 \%$ for any radiotherapy and $20 \%$ to $49 \%$ for any active treatment. One-year patient survival ranged from $11 \%$ to $34 \%$. There was evidence of health authority level variation even after adjusting for case mix. Patients resident in health authorities with higher age standardised lung cancer incidence were less likely to receive active treatment. Patients whose first hospital attendance was at a radiotherapy centre were more likely to receive active treatment (OR $1.58,95 \% \mathrm{Cl}$ 1.16 to 2.15$)$, chemotherapy $(1.38,1.05$ to 1.80$)$ or radiotherapy $(1.86,1.28$ to 2.71$)$. There was some evidence that patients whose first hospital attendance was at a radiotherapy centre survived longer, but this was inconsistent between models.

Conclusions: There is geographical inequality in the treatment given to lung cancer patients and patient survival in South East England. There was some evidence to suggest that these inequalities might be explained by variations in access to oncology services. Future studies should investigate the pathways and barriers to specialist care in this condition.

\section{Mental health II}

\section{THE SOCIAL DETERMINANTS OF HOSPITAL TREATED PARASUICIDE: EARLY FINDINGS FROM THE NATIONAL PARASUICIDE REGISTRY IRELAND}

C. Thunhurst, P. Corcoran, I.J. Perry. Department of Epidemiology and Public Health, University College Cork, Cork, Republic of Ireland

Objective: To utilise early findings from the National Parasuicide Registry Ireland and the preceding monitoring study to explore the social patterning of hospital-treated parasuicide within the Cities of Limerick and Cork.

Design: Primary data from the National Parasuicide Registry Ireland and the preceding monitoring study are analysed against secondary data from the most recent Irish full census and mid-term census to examine the social patterning of hospital-treated parasuicide within two major cities in Ireland.

Setting: The National Parasuicide Registry Ireland constitutes the first national attempt at whole population monitoring of parasuicide. The Registry has been established progressively throughout 2001. The Registry employs methods of data collection that were previously employed in a monitoring study of two health boards undertaken from 1995 to the end of 2000. Data from the first four health boards covered by the national Registry are available from 1 January 2001.

Participants: All incidents of hospital-treated parasuicide presented to major Accident and Emergency Departments in four health boards in the Republic of Ireland, with further exploration of those from the Cities of Limerick and Cork. 
Results: The incidence of parasuicide in four Irish Health Boards is presented showing variation by gender, age, method and area. The overall incidence rate is 116 episodes per 100,000 population, and varies six-fold from 57 for Waterford County to 340 for Limerick City. Parasuicide in Ireland exhibits significant geographical variation, with particularly elevated incidence in some urban areas. The two major cities within those four health boards are examined further to allow fuller exploration of the association between the incidence of parasuicide and area level deprivation markers. Simple one-variable and composite multi-variable indices are used, with a best-fit model explaining 57 per cent of variation. Multivariate clustering methods are employed to produce a typology of small urban areas. When person-based parasuicide rates are analysed by cluster, a three-fold variation emerges, from 147 to 427 per 100,000 population.

Conclusions: Parasuicide is an important indicator of population health. Early findings from the National Parasuicide Registry Ireland and the substantive data available from the preceding monitoring study indicate that a rich picture of social patterning contributes to an understanding of parasuicide. The availability of large area and small area data from the 2002 national census of Ireland will enable a fuller exploitation of data from the National Parasuicide Registry Ireland permitting more directed targeting of preventive services to be undertaken.

\section{DEATHS FROM ANTIDEPRESSANTS IN ENGLAND AND WALES 1993 TO 1997: ANALYSIS OF A NATIONAL DATABASE OF DRUG-RELATED POISONING DEATHS}

A. Baker', R. Shah², Z. Uren', A. Majeed ${ }^{3}$. 'Office for National Statistics, London; '2Sutton Hospital, Surrey; ${ }^{3}$ School of Public Policy, University College London, London

Objective: To examine trends in the mortality associated with overdoses and poisoning with antidepressants, and to compare the relative mortality associated with different antidepressants in routine clinical practice.

Design: Analysis of a national database of deaths from overdose and poisoning, containing all deaths with an underlying cause regarded by the Office for National Statistics as being drug related. Every mention of a substance recorded on the death certificate, or mentioned by the coroner, is held, and all prescription drugs are coded to British National Formulary (BNF) categories. Information on the number of prescriptions for individual antidepressants dispensed in the community in England during 1993-1997, obtained from the Department of Health.

Setting: England and Wales, 1993-1997 (England only for analysis by prescription numbers.)

Main outcome measures: Age and sex specific death rates associated with overdose and poisoning with antidepressants; numbers of deaths per 100,000 prescriptions of individual antidepressants.

Results: $20 \%$ (2503) of all deaths from overdose or poisoning were antidepressant related. The number of antidepressant related deaths increased by $18 \%$ between 1993 to 1997 , from 459 to 539 . $95 \%$ of deaths were associated with tricyclic antidepressants and two antidepressants, dothiepin and amitriptyline, were associated with $82 \%$ of all antidepressant related deaths. Tricyclic antidepressants were associated with 5.3 deaths per 100,000 prescriptions, compared with 4.4 for mono-amine oxidase inhibitors, and 0.4 for selective serotonin reuptake inhibitors and related antidepressants. Annual death rates were highest in men aged $30-44$ years $(18.2$ per million) and women aged $45-59$ years (14.8 per million). Death rates from antidepressants were 2.5 times higher in the most deprived fifth than in the least deprived fifth of enumeration districts. Newly available data for 1998 to 2000 will also allow the presentation of results to be extended for those years.

Conclusions: Antidepressants are an important cause of death from poisoning and overdose. The selective serotonin reuptake inhibitors and the other newer antidepressants are associated with less than $10 \%$ of the risk of death than the older antidepressants. There is also a very strong association between area deprivation and deaths from antidepressants. Deaths from antidepressant overdose could be cut substantially by reducing the number of prescriptions for the older antidepressants, particularly to those in high risk groups.

\section{CROSS SECTIONAL STUDY TO ASSESS THE BURDEN OF MENTAL DISORDER IN YOUNG OFFENDERS IN A DORSET PRISON}

J. Parkes', D. Jenkins'2, J. Goddard', S. Bennett'. 'Health Care Research Unit Southampton General Hospital; '2Public Health Department Dorset Health Authority

Background: A health needs assessment performed within the Dorset prisons, has identified mental illness of young offenders as a priority.
To inform recommendations for improvement in health care the burden of illness needs to be known.

Aims: To ascertain prevalence of mental disorder in young offenders in HMP YOI Portland.

Objectives: To conduct a cross- sectional survey in prisoners aged 15 to 20 years to assess the burden of neurosis, psychosis, personality disorders, hazardous drinking and substance abuse.

Methods: The study was conducted using structured interviews and utilising a questionnaire based on validated tools. Analysis was performed using SPSS and included comparisons of the prevalence of mental disorder in the prison to a similar age cohort in the general population, to sentenced prisoners in older age groups, and determination of any correlation between demographic factors such as age, looked- after status, and mental disorder.

Results: The coverage was $94 \%$. The prevalence of mental illness in sentenced male young offenders is very high with $95 \%$ having at least one mental disorder. Prisoners in this study were twice as likely to have a neurotic illness than that those similar aged men in the general population, two and a half times as likely to have a psychotic illness, three times as likely to exhibit hazardous drinking, eleven times as likely to have used illicit drugs, five times as likely to be drug dependent and twice as likely to have personality disorder. In particular, deliberate self-harm and suicidal ideation is worryingly high in this population. Use of services both in and out of prison is far less than the need expressed by this burden of disease. Socio-demographic data confirm that many of the young prisoners have poor experience of secondary education, early contact with both the judicial system and illicit drug use.

Conclusions: The burden of mental disorder in young prisoners is large with increased risk of all mental disorders assessed. There is low uptake of helping services in and out of prison

Effective services both in and out of prison are urgently needed to provide effective management of the mental health needs of this vulnerable and traditionally hard to reach population.

\section{Sexual behaviour and HIV}

\section{LEARNING ABOUT SEX: RESULTS FROM NATSAL 2000}

W. MacDowall', K. Wellings', K. Nanchahal', C. Mercer², B. Erens ${ }^{3}$, K. Fenton', A. Johnson'. 'Sexual Health Programme, Health Promotion Research Unit, London School of Hygiene \& Tropical Medicine, Keppel Street, London WC IE 7HT; ${ }^{2}$ Department of Sexually Transmitted Diseases, Royal Free and University College Medical School, Mortimer Market Centre, off Capper Street, London WCIE 6AU; ${ }^{3}$ National Centre for Social Research, 35 Northampton Square, London ECIV OAX

Objectives: To describe sources of information on sexual matters, adequacy of information, additional information needs and preferred source. Further, to examine the associations between source of sex education and expressed need for further information.

Subjects and design: The second National Survey of Sexual Attitudes and Lifestyles (Natsal 2000) is a probability sample survey conducted in 1999-2001 of 4762 men and 6399 women aged 16-44 living in Britain. Respondents were asked a number of questions regarding learning about sexual matters, including sources of information, what they would like to have know more about and from whom.

Main outcome measures: Main source and adequacy of information, additional information needs and preferred source. Expressed information needs were grouped into three categories: biological, risk reduction and psychosexual.

Results: School lessons are now the leading source of information about sexual matters among young people, being the main source for $39.2 \%$ men and $30.1 \%$ women aged $16-19$. Parents and school were the preferred source of further information for over two-thirds of respondents. In contrast to the very small proportion $(<1 \%)$ who cite 'doctor, nurse or clinic' as their main source, $7.3 \%$ men and $7.7 \%$ women aged 16-19 would like to have got further information from them. Amongst sexually experienced 16-19 year olds, $73.3 \%$ men and $76.9 \%$ of women reported that they did not have enough information about sexual matters. The proportion of men expressing a need for additional biological information at the time when they first felt ready to have some sexual experience was twice as high among 30-44 year olds than amongst those aged $16-19$ (32.6\% and $15.6 \%$ respectively), while the proportion reporting wanting to have known more about risk reduction has remained constant (around 50\%) across the age groups. Older respondents were more likely to report wanting to have known more about psychosexual aspects $160.5 \%$ of $30-44$ 
year old men compared with $45.6 \%$ of $16-19$ year olds). Similar patterns were observed among women. Those reporting parents as their main source of information were least likely to have wanted further information.

Conclusions: School lessons are now the main source of information about sexual matters among young people. However, three-quarters of respondents expressed a need for further information at the time that they first felt ready to have sexual experience. Although advances have been made in providing biological information, these data suggest that there has been no change in perceived adequacy of knowledge on risk - reduction.

\section{EVALUATION OF BAR AND GYM BASED PEER-LED SEXUAL HEALTH INTERVENTIONS FOR GAY MEN IN GLASGOW AND LONDON}

G. Hart', J. Elford', L. Williamson', G. Bolding', L. Sherr'. 'MRC Social \& Public Health Sciences Unit, University of Glasgow; ${ }^{2}$ Institute of Health Sciences, School of Nursing and Midwifery, City University, London; ${ }^{3}$ Department of Primary Care \& Population Sciences, Royal Free \& University College London Medical School, London

Objectives: Employing a diffusion of innovation model, peer-led HIV prevention programmes were developed for gay men in bars in Glasgow and gyms in central London. We sought to evaluate the impact of community-based peer-led HIV prevention initiatives among gay men in the two cities.

Design: Two controlled trials

Data sources: Data were collected by means of self administered questionnaires distributed at baseline and follow up in the gay bars of Glasgow and Edinburgh, and in 5 gyms in Central London.

Main outcome measures: Reduction in reported unprotected intercourse (Glasgow, London); increased uptake of sexual health services (Glasgow).

Results: In Glasgow (intervention city) and Edinburgh (control city), 2276 men participated at baseline (1996) and 2498 at outcome (1999), with an average $78 \%$ response rate. The intervention did not produce community-wide changes in sexual behaviours. The only significant intervention effects were amongst men who had direct contact with the intervention, with higher uptake of Hepatitis $B$ vaccination and HIV testing. In London, from September 1997 to April 1999, at baseline and at 18 month follow up, the proportion of men $(n=1000)$ reporting high risk sexual behaviour remained constant (14\%) while there was an increase from $73 \%$ to $79 \%$ in the proportion ever-tested for HIV. No significant differences were found between intervention and control gyms in the rate of change of any of these outcome variables at follow up.

Conclusions: Peer education was not an effective tool for sexual behaviour change among gay men in bars in Glasgow or central London gyms. In Glasgow the intervention was successful amongst those men with whom there was direct contact in increasing the uptake of sexual health services, but there was no evidence of 'diffusion of innovation'. Peer education for safer sex may be inappropriate for UK gay men at this stage of the HIV epidemic, although it could prove useful in increasing access to sexual health services. Implications for the sexual health strategies for England and Scotland will be discussed.

\section{HIV/AIDS PEER EDUCATION IN THE SOUTH AFRICAN WORKPLACE: IMPACT ON PERCEPTION OF PERCEIVED RISK}

N. Sloan', T. Davidson'2, J.E. Myers ${ }^{3}$. 'Sowerby Centre for Health Informatics, University of Newcastle; ${ }^{2}$ Health Services, WoolWorths Pty Ltd, Cape Town, South Africa; ${ }^{3}$ School of Public Health \& Primary Health Care, University of Cape Town, South Africa

Aim: To determine the relationship between HIV peer education programmes in the workplace and level of self perceived risk of acquiring HIV. This is part of a wider evaluation of an HIV peer education programme that was conducted by comparing two South African retail companies, one with an HIV peer education programme and one without.

Methods: A total of 900 questionnaires was completed by employees of two South African retail companies from three regions (Gauteng, Western Cape and Eastern Cape/ KwaZulu-Natal) over a period of six months (Feb-July 2001). Questionnaires were made available in English, isiZulu, isiXhosa, Afrikaans, SiSwati, Southern Sotho and Northern Sotho; and completed during in-store training sessions after the purpose of the study was explained and consent was obtained. The questionnaire tests level of knowledge about HIV/AIDS, attitude towards people living with HIV/AIDS, level of condom use and level of attendance at HIV peer education sessions at work (if applicable). In addition, the questionnaire asked participants to score how at risk they felt of acquiring HIV on a Likert scale from $0-10$. Participants were then invited to explain their score. Logistic regression modelling was used to analyse the relationship of perceived risk $(<5,>=5)$ with demographic factors, level of knowledge about HIV, attitude towards people with HIV, condom use and attendance HIV education sessions. The qualitative findings drawn from the participant's explanation of their perceived risk score was analysed for relevant themes.

Results: The majority of participants thought they were at low perceived risk for acquiring HIV (mean perceived risk score of 2.5 out of a possible 10). Significant factors that explain the $7 \%$ of variance in perceived risk were age, sex, company, area and relationship status. Qualitative findings showed that people often knew they were undertaking high risk behaviour (e.g. multiple partners and not using condoms) but still felt they were at low perceived risk (i.e. score of 4 or less). Participants who did score themselves at high perceived risk (i.e. 5 or above) offered a variety of explanations from lack of trust in condoms to the strong probability of having sex with someone who is HIV positive because of the high prevalence of HIV in South Africa. The majority of married participants felt they were at no or low perceived risk because they had only one sex partner.

Conclusion: The findings indicate that education alone does not impact on perceptions of perceived risk and therefore such programmes may not be effective in causing change in from high risk to low risk behaviour.

\section{Miscellaneous}

\section{A SURVEY INTO THE QUALITY, CONTENT AND STYLE OF CURRENT EPIDEMIOLOGICAL PUBLICATIONS}

T. Collier', S. Pocock', K. Dandreo ${ }^{2}$, B De Stavola', M. Goldman². L. Kalish', L. Kasten'2, V. McCormack'. 'London School of Hygiene and Tropical Medicine, London, UK; ${ }^{2} \mathrm{New}$ England Research Institute, Boston, USA

Epidemiological studies vary enormously in the quality, content and style of their publications. There is a need for guidelines to aid authors and journals in raising the standards of such published observational research in human populations. As an enquiry into the current state-ofthe-art in published epidemiologic research, we identified all epidemiological studies published in English in January 2001 in major epidemiological journals, major general medical journals and a sample of specialist medical journals. Eligible studies were those investigating the association between exposure variable(s) and disease outcome(s) in individual subjects from the general population. Purely hospital-based studies were excluded.

73 such publications were identified: 37 cohort studies, 25 case-control studies, 9 cross-sectional studies and 2 others. The great majority related to fatal or non-fatal disease events, though 9 studies concerned quantitative disease measures. The majority of exposure items were quantitative measures, but in the main these data were grouped and presented in several ordered categories.

The extent of adjustment for confounding variables varied enormously. 22 studies used matching (e.g. of cases and controls) while 67 studies employed some confounder-adjusted analysis: the median number of confounders adjusted for was 7 . For many studies it was unclear how and why the confounders were selected.

As regards statistical analyses, the odds ratio was the most commonly employed measure of the exposure-disease outcome association (40 studies), while a further 27 studies (mostly cohort studies) used hazard ratios or rate ratios. All other forms of statistical result were relatively uncommon. 69 studies used confidence intervals, 38 studies used P-values with only 2 studies using neither method of inference. One journal (Epidemiology) did not permit P-values. 52 studies undertook subgroup analyses in order to detect potential effect modifiers; 32 of these studies then made claims that such effect modifiers did exist. Many of these studies did not make appropriate subgroup inferences (e.g. interaction test), so that many of these claims may not be justified. Studies varied considerably in size, and further investigation will be undertaken into whether studies tended to have adequate statistical power. We suspect many did not.

While only a few of these studies could be deemed post hoc not to merit publication, there are clearly many facets of study design, conduct, analysis and reporting which could be substantially improved by authors and the journals' editorial and refereeing policy. We hope this survey acts as a stimulus to raising the standards of epidemiological publications. 


\section{THE IMPACT OF EDITORIAL GUIDELINES ON THE CLASSIFICATION OF RACE/ETHNICITY IN THE BRITISH MEDICAL JOURNAL}

G.T.H. Ellison, M. Rosato. Social Science Research Unit, Institute of Education, University of London, London

Background: In 1996 the BM introduced "guidelines on the use of ethnic, racial, and cultural descriptions in published research". These proposed that the categories used should "relate to the type of hypothesis under investigation" and that authors should "describe the logic behind their 'ethnic groupings'". The guidelines specifically recommended that authors use: "accurate descriptions rather than catch-all terms" to identify the individuals or populations examined; terms that "reflect how the groups were demarcated"; and a range of information on ethnicity, race and culture whenever it is unknown which is the "most important influence".

Methods: To assess whether the guidelines had any impact on the classification and use of race/ethnicity in published research, the journal's online archive was searched for any articles classified as 'papers' containing the terms 'race or racial or ethnic *' which had been published between $1^{\text {st }}$ January 1994 and $31^{\text {st }}$ December 2000. Any geographical, phenotypic, cultural, physiognomic or census labels used to classify race/ethnicity were recorded and additional searches were conducted for papers which had used one or more of these labels without race/ethnicity. Content analyses were subsequently conducted on both sets of papers to establish whether the categories were used as descriptive, sampling, control and/or explanatory variables, and whether their use complied with the 1996 guidelines.

Results: 68 different labels were found in the 201 papers that mentioned race/ethnicity, and additional searches on these labels located a further 213 papers for inclusion in the content analyses. Very few of the papers explicitly described why race/ethnicity was considered relevant to their hypothesis or analyses, and this made it difficult to assess whether appropriate categories had been used. Even after the guidelines had been introduced, few of the papers explained why they had chosen to use particular categories, and none consistently used descriptive labels or terms that indicated how these categories had been applied. Only a few papers explicitly set out to explore racial/ethnic disparities in health, and none of these measured a comprehensive range of cultural, socio-economic and genetic variables.

Conclusion: The classification of race/ethnicity in papers published by the British Medical Journal remains haphazard and poorly documented despite the introduction of editorial guidelines. While it is clear that the guidelines have not been followed, it is not clear whether they were ignored or were impossible to enforce. Further research is required to assess the acceptability and feasibility of such guidelines amongst editorial staff, manuscript reviewers and authors.

\section{GLAD TO BE GREY: A RESTATEMENT OF THE HYPOTHETICO-DEDUCTIVE MODEL}

K. Hopayian. School of Medicine, Health Policy and Practice, University of East Anglia, Norwich

Background: Science in general and medical science in particular have come under increasing criticism for being incapable of providing a complete model of nature, health and health care. Criticism has arisen from several quarters: qualitative research, postmodernism, and complexity theory. Common themes run through all these, namely, accusations that science and medical science are limited by reductionism, linear thinking, and positivism. At its worst, science is accused of a black/white view of the world. In contrast, alternative models are promoted as having better explanatory power or understanding of complex phenomena.

Aims: To stimulate a debate on the epistemology of medicine in order to inform research, education, and practice.

Methods: Recent publications in English language general medical journals will be examined to demonstrate the case made against science and to judge the validity of the accusations.

A brief survey of alternative philosophical theories of the scientific process, including from scientists themselves, will be presented.

This will demonstrate that science is best seen as an activity that does not view the world as black/white but constantly seeks to describe shades of grey.

The value of the hypothetico-deductive model, drawn largely on the work of Karl Popper, will be re-examined and its superiority in explaining the search for truth demonstrated.
Conclusions: Some of the proposed alternatives occasionally stray towards inductionism, thereby falling into the trap that the hypothetico-deductive model avoids. The hypothetico-deductive model is the best guarantee we have for the progress of science, medical science, and health care.

\section{Methods}

\section{FASTING MEASUREMENTS IN EPIDEMIOLOGICAL STUDIES: AN UNRECOGNISED SOURCE OF BIAS}

J.R. Emberson', P.H. Whincup ${ }^{2}$, M. Walker', M. Thomas', K.G.M.M. Alberti'. 'Department of Primary Care and Population Sciences, Royal Free Hospital, London; ${ }^{2}$ Department of Public Health Sciences, St. George's Hospital Medical School, London; ${ }^{3}$ Department of Chemical Pathology, Royal Free Hospital, London; ${ }^{4}$ Department of Diabetes and Metabolism, University of Newcastle on Tyne, Newcastle

Background: Epidemiological studies generally aim to make simple but unbiased measurements of individuals. For this reason measurements of metabolic variables (including blood lipids, glucose and insulin) are usually carried out after a period of fasting, and it is usually assumed that such measurements are unbiased. We examined whether the use of a defined protocol in a cardiovascular survey abolished the influence of fasting duration and time of day on biochemical measurements.

Methods: Cross-sectional survey of British Regional Heart Study participants (4252 men aged 60-79 years), in which men without diabetes were asked to provide a blood sample after fasting for at least 6 hours. Serum total, HDL and LDL cholesterol, triglyceride and insulin, and plasma glucose concentrations were measured between $0800 \mathrm{~h}$ and $1800 \mathrm{~h}$.

Results: Men who did not fast for 6 hours had lower mean LDL cholesterol and higher glucose, insulin and triglyceride levels than fasting men; these differences were more marked among diabetics. Among fasting men without diabetes, total and HDL cholesterol levels displayed no consistent relation to fasting duration or time of day. In contrast, mean insulin and glucose levels were strongly related to time of day, falling gradually throughout the morning and remaining stable in the afternoon. They showed a periodic relation with fasting duration above 6 hours, a pattern that was abolished after adjustment for time of day effects. However, the associations with time of day were unaffected by adjustment for fasting duration. Mean triglyceride levels in fasting men without diabetes fell with increasing fasting duration, and rose gradually through the day; the effect of fasting duration was abolished after adjustment for time of day. Adjustments to compensate for variations due to both fasting duration and time of day were performed.

Conclusion: Biochemical measurements from fasted subjects should not necessarily be accepted as unbiased measurements of individuals. Marked variations with fasting duration and particularly with time of day may still occur. In the analysis of metabolic measurements in epidemiological studies, it is important to examine the extent of variation by time of day and fasting duration, and to adjust for them as appropriate. Ideally, future studies should aim to standardise both the period of fasting and the time of day at which tests are performed.

\section{MISSING, MEDIOCRE OR MERELY OBSOLETE? AN EVALUATION OF UK DATA SOURCES FOR CORONARY HEART DISEASE}

B. Unal Aslan, J. Critchley, S. Capewell. Department of Public Health, University of Liverpool

Introduction: Policy decisions on health and health care require good evidence, particularly since resources are limited. Coronary heart disease (CHD) is the most common cause of death in the UK, yet currently there is no single comprehensive source of information to support strategies for CHD prevention and treatment.

Objective: To evaluate the availability and quality of UK CHD data sources since 1981 .

Methods: We identified and appraised data for England and Wales on:

a) CHD patient numbers (including acute myocardial infarction, secondary prevention, angina, heart failure and hypertension), b) uptake of medical and surgical treatments, c) effectiveness of specific cardiological treatments, and d) population trends in major cardiovascular risk factors (including smoking, cholesterol, hypertension, obesity, diabetes, physical activity and deprivation). 
Results: The main data sources identified from papers, reports and web sites and appraised were: Population and patient data - easily accessible from ONS official statistics \& BHF Annual CHD Statistics; Population based risk factor data, principally from the British Regional Heart Study, General Household Survey (GHS), \& the Health Survey for England (HSE)- limited for 1981, but more extensive by 2000. For cholesterol, limited data were available for the early 1980s, while changing laboratory methods used in the HSE complicate interpretation of recent trends. Blood pressure data were likewise limited until recently. Although there was indirect evidence of declines in physical activity, no comprehensive population-based measures were available before 1990. However, good data for trends in smoking prevalence are available from GHS. Hospital admissions information since 1998 was available online from Hospital Episode Statistics. However trend data, and details of hospital interventions were very limited. At primary care level, limited prescribing and uptake data were available from PACT (Prescription Analysis and Cost), audits and some studies.

In general, data for women and the elderly (over 65) were particularly scarce. Good efficacy/ effectiveness data on clinical interventions were generally available from meta-analyses and randomised trials.

Conclusions: Every year in Britain, CHD causes over 110,000 deaths and costs over $£ 10$ billion. However, information on CHD is fragmented, patchy and mixed in quality. Future CHD disease monitoring and evaluation of interventions will require comprehensive and accurate population-based information on trends in treatment uptake and risk factors. The integration of existing data recommended by the recent UK CHD Information strategy will be valuable. However, regular and comprehensive population-based surveys (including women and elderly people), using standardised methodology will also be essential.

\section{EXPLORING THE RELATIONSHIP BETWEEN SELF-RATED HEALTH, GLOBAL QUALITY OF LIFE, AND SPECIFIC DOMAINS OF QUALITY OF LIFE}

D. Buck', A. Jacoby', G.A. Ford'2. 'Department of Primary Care, University of Liverpool; 'Wolfson Unit of Clinical Pharmacology, University of Newcastle upon Tyne
Background and aim: The terms 'health status' and 'quality of life' (QOL) are often used interchangeably. However, a recent meta-analysis demonstrated that the two appear to be distinct constructs. In particular, mental health domains were found to have a far greater impact than physical functioning on global ratings of QOL. Conversely, physical functioning domains were more influential than dimensions of mental health on self-rated health. This presentation will report the findings from a new study that further examined the relationship between these concepts.

Methods: Informants (stroke survivors) rated their overall health and overall QOL on a 5-point scale. The relationship between these global variables and informants' mean scores on 11 specific QOL domains was examined. Specific domains and informants' age, gender and area of residence were entered into a stepwise regression analysis with the global items as the dependent variables.

Results: Scores on 6 of the 11 specific QOL domains (Mobility, ADL/Self-Care, Cognition, Sleep, Fatigue, Feelings) were significantly associated with self-rated health. Scores on all but one domain (Vision) were significantly associated with ratings of overall QOL. High scores (i.e. greater perceived impact) on Sleep and Vision domains were the strongest predictors of lower self-rated health. High scores on Feelings and Sleep domains were the strongest predictors of lower global QOL.

Conclusion: These findings shed further light on the much-used concept of perceived health status. Although they lend some support to the notion that perceived health status and QOL may be distinct constructs, the evidence is far from conclusive. For example, issues around the concept of Sleep seem to reflect both physical and mental well-being. Sleep emerged as a predictor, albeit not the strongest, of global QOL. Moreover, moderately high correlations were found between self-rated health and global QOL. It is possible that individuals' conceptualisations of the terms 'quality of life' and 'overall health' vary depending not only on current health status but also on the nature of any illness or condition they may have. Further quantitative and qualitative work in this area, including with people with no health problems, would help to clarify the issue. This is important since global items such as those described here are often used to determine the validity of more lengthy health outcome measures. 